ARTICLE

DOI: $10.1038 / \mathrm{s} 41467-017-02786-5$

\title{
RASSF1A uncouples Wnt from Hippo signalling and promotes YAP mediated differentiation via p73
}

\author{
Angelos Papaspyropoulos ${ }^{1}$, Leanne Bradley ${ }^{1}$, Asmita Thapa ${ }^{1}$, Chuen Yan Leung ${ }^{2,3}$, Konstantinos Toskas ${ }^{1}$, \\ Delia Koennig ${ }^{1}$, Dafni-Eleftheria Pefani ${ }^{1}$, Cinzia Raso ${ }^{4}$, Claudia Grou${ }^{1}$, Garth Hamilton ${ }^{1}$, Nikola Vlahov ${ }^{1}$, \\ Anna Grawenda ${ }^{7}$, Syed Haider ${ }^{5}$, Jagat Chauhan ${ }^{5}$, Ludovico Buti ${ }^{6}$, Alexander Kanapin (1) 7 , Xin Lu' \\ Francesca Buffa (10 5 , Grigory Dianov ${ }^{1,8}$, Alex von Kriegsheim ${ }^{4}$, David Matallanas ${ }^{4}$, Anastasia Samsonova ${ }^{7}$, \\ Magdalena Zernicka-Goetz ${ }^{2,3}$ \& Eric O'Neill (1) ${ }^{1,4}$
}

Transition from pluripotency to differentiation is a pivotal yet poorly understood developmental step. Here, we show that the tumour suppressor RASSF1A is a key player driving the early specification of cell fate. RASSF1A acts as a natural barrier to stem cell self-renewal and iPS cell generation, by switching YAP from an integral component in the $\beta$-catenin-TCF pluripotency network to a key factor that promotes differentiation. We demonstrate that epigenetic regulation of the Rassf1A promoter maintains stemness by allowing a quaternary association of YAP-TEAD and $\beta$-catenin-TCF3 complexes on the Oct4 distal enhancer. However, during differentiation, promoter demethylation allows GATA1-mediated RASSF1A expression which prevents YAP from contributing to the TEAD/ $\beta$-catenin-TCF3 complex. Simultaneously, we find that RASSF1A promotes a YAP-p73 transcriptional programme that enables differentiation. Together, our findings demonstrate that RASSF1A mediates transcription factor selection of YAP in stem cells, thereby acting as a functional "switch" between pluripotency and initiation of differentiation.

\footnotetext{
${ }^{1}$ CRUK/MRC Oxford Institute, Department of Oncology, University of Oxford, Oxford OX3 7DQ, UK. ${ }^{2}$ The Wellcome Trust/Cancer Research UK Gurdon Institute, University of Cambridge, Cambridge CB2 1QN, UK. ${ }^{3}$ Department of Physiology, University of Cambridge, Cambridge CB2 3EG, UK. ${ }^{4}$ Systems Biology Ireland, University College Dublin, Dublin 4, Ireland. ${ }^{5}$ Applied Computational Genomics, Department of Oncology, University of Oxford, Oxford OX3 7DQ, UK. ${ }^{6}$ Ludwig Institute for Cancer Research, Nuffield Department of Clinical Medicine, University of Oxford, Oxford OX3 7DQ, UK. ${ }^{7}$ Bioinformatics Research Core, Department of Oncology, University of Oxford, Oxford OX3 7DQ, UK. ${ }^{8}$ Institute of Cytology and Genetics, Russian Academy of Sciences, Lavrentyeva 10, Novosibirsk 630090, Russian Federation. Correspondence and requests for materials should be addressed to E.O'N. (email: eric.oneill@oncology.ox.ac.uk)
} 
W NT, Notch, Hedgehog and Hippo signalling pathways play important roles in maintaining pluripotency ${ }^{1}$. The transcriptional co-factors of the Hippo pathway, YAP and TAZ (Wwtr1) promote stem cell self-renewal and pluripotency by mediating TEA-domain (TEAD1-4) transcription. Of the TEA domain family of transcription factors, TEAD2 is upregulated in embryonic stem cells (ESC) ${ }^{2}$ with TEAD2 and TEAD4 expressed early in embryogenesis ${ }^{3,4}$. YAP-TEAD transcriptional complexes directly activate Pou5f1 (Oct4; hereafter Oct4) expression and the subsequent expansion of pluripotent cells 5 . YAP plays a crucial role in cell specification of developing embryonic blastocysts, where in line with studies in ESCs, YAP is also nuclear and drives pluripotency in the inner cell mass (ICM) 6 . However, definition of the trophectoderm (TE) depends on YAP-mediated expression of $\mathrm{CDX}^{4}$. This dichotomous role of YAP in the blastocyst has been suggested to be due to transcriptional cooperation with $\beta$-catenin in the ICM, but also suggests that YAP may have distinct roles in pluripotent versus differentiating cells, such as the TE. Accumulating evidence supports roles for YAP and TAZ in early ESC differentiation and terminal differentiation of tissues, guided by distinct transcription factor selection ${ }^{7-10}$. The WNT pathway effector TCF1/LEF can also actively repress or activate expression of Oct4, depending on $\beta$-catenin association. In this manner, $\beta$-catenin plays a major role as a mediator of WNT signaling in maintaining stem cell selfrenewal $^{11,12}$ but requires YAP to drive dedifferentiation of cardiac progenitors ${ }^{13}$, indicating that WNT and Hippo converge to regulate pluripotency.

The Hippo pathway is a signal transduction cascade where the MST1/2 kinases activate LATS/NDR kinases to phosphorylate YAP/TAZ. SAV1 and RASSF1-6 are direct binding scaffolds of MST1/2 that regulate and localise pathway activation and YAP activity. LATS/NDR-mediated phosphorylation of YAP on five serine residues prevents association with TEADs and the pSer127-YAP (Ser112 in mouse) site presents a binding site for 14-3-3 proteins that increases cytoplasmic retention ${ }^{14}$. During differentiation of ESCs, activation of Hippo signalling restricts TEAD-mediated transcription of Oct4, however, exactly how the Hippo pathway is activated during differentiation is not known ${ }^{5}$.

While the pathways regulating pluripotency are well understood, those driving early stages of transition to a differentiated state remain elusive. The earliest specification of stem cell fate upon exiting pluripotency has recently been reported to be dependent on $\mathrm{p}^{7} 3^{15}$, a transcriptional partner of the Hippo pathway mediator YAP, that is also required during terminal differentiation ${ }^{16-18}$. Activation of the Hippo pathway, resulting in increased pSer127-YAP, is also required for optimal association with $\mathrm{p} 73^{19-22}$. Moreover, RASSF1A-mediated Hippo pathway activation promotes both pS127-YAP and the formation of active YAP-p73 transcriptional complexes, while loss of RASSF1A expression allows the formation of pro-proliferative YAP-TEAD ${ }^{21,23,24}$. RASSF1A silencing via promoter hypermethylation is widespread in solid malignancies and correlates with a loss of pS127-YAP, elevated YAP/ $\beta$-catenin mediated transcription and increased ESC signatures in tumors ${ }^{25-27}$. Furthermore, human mesenchymal stem cells harbouring targeted promoter methylation of RASSF1A display oncogenic properties and maintain stemness ${ }^{28}$. Thus, methylation of RASSF1A is proposed to induce dedifferentiation of tumour cells, which may account for poor prognosis of patients whose cancers are associated with high levels of epigenetic silencing ${ }^{29,} 30$.

A potential role for RASSF1A in early development may be indicated as methylation of the Rassf1A promoter is observed in both mouse and human oocytes/zygotes and decreases as cells pass through 2-8 cell stages of the pre-implantation embryo, in line with increased mRNA expression ${ }^{31}$. These events occur inversely to the methylation of Nanog and Oct4 during the epigenetic reprogramming of the embryo and point to a more sophisticated regulation on the epigenome during embryogenesis. As frequently observed for regulatory components of the preimplantation embryo and most recently demonstrated for p73, 32, genetic ablation of individual Hippo components (e.g., Mst1, Mst2, Rassf1) in mice can demonstrate mild or late phenotypes due to redundancy and compensation. Importantly in humans, epigenetic silencing of RASSF1A expression is associated with developmental defects in the placenta, pointing to an underappreciated role in early development ${ }^{33}$.

Here, we provide evidence that RASSF1A regulation of the Hippo pathway effector YAP controls the balance between pluripotency and differentiation of ESCs. We provide evidence that YAP-TEAD2 and $\beta$-catenin-TCF3 cooperate on the Oct4 promoter to promote expression and pluripotency under stem cell maintenance conditions. Differentiation induces expression of RASSF1A, removing YAP from the TEAD2 complex and disabling Oct4 transcription. We find that RASSF1A-induced pS127-YAP is not inactive but plays an active role in differentiation through direct binding to p73 and activation of lineage specification genes.

\section{Results}

RASSF1A is expressed in differentiating stem cells. Epigenetic regulation of genes has been widely implicated in stem cell fate specification $^{34}$. RASSF1A expression is widely observed to be epigenetically regulated in cancer, and demethylation of the human RASSF1A promoter has been shown to accompany differentiation of neural progenitors into somatic cells ${ }^{35}$. To determine whether RASSF1A played a role in stem cell differentiation we first examined levels of RASSF1A in human ( $\mathrm{H} 1$ and $\mathrm{H} 9$ ) and mouse (J1 and V6.5) ESC lines allowed to differentiate over a period of 7-14 days. hRASSF1A and mRassf1A (hereafter Rassf1A) mRNA expression increased upon differentiation of both human and mouse ESCs (Fig. 1a, b). Using the ENCODE Project database to compare human ESC and mesoderm-derived progenitors, we find the RASSF1 promoter appears in a 'poised' epigenetic state in ESCs rather than completely silenced, as the promoter is marked with both activating $\mathrm{H} 3 \mathrm{~K} 4^{\mathrm{me} 2}$ and repressive $\mathrm{H} 3 \mathrm{~K} 27^{\mathrm{me}}$ histone modifications (Supplementary Fig. 1a). Differentiation into mesoderm progenitors is coincident with loss of the repressive mark specifically at the RASSF1 promoter $\mathrm{CpG}$ island, conversion of $\mathrm{H} 3 \mathrm{~K} 4^{\mathrm{me} 2}$ to $\mathrm{H} 3 \mathrm{~K}^{\mathrm{me}}{ }^{\mathrm{m}}$ (a mark of transcriptional activity) and increased transcription (Supplementary Fig. 1a). To confirm changes in endogenous expression, a pool of E14Tg2a mESC were allowed to terminally differentiate in the absence of LIF (-LIF), indicated from the existence of beating cardiac progenitor cells (Fig. 1c, Supplementary Fig. 1b and Supplementary Movie 1). Levels of both endogenous RassfiA mRNA and protein were elevated in differentiated (-LIF) cells compared to the undifferentiated (+LIF) cells (Fig. 1c). Moreover, we find reduced $\mathrm{CpG}$ island promoter hypermethylation and increased active transcription $\mathrm{H} 3 \mathrm{~K} 4^{\mathrm{me} 3}$ chromatin marks at the RASSF1A promoter in differentiated versus pluripotent mouse ESCs (Fig. 1d, e and Supplementary Fig. 1a). Thus, demethylation of RassfiA along with elevated mRNA expression occurs when cells move from a pluripotent state towards a more terminally differentiated fate at the same time as expression of pluripotency genes Nanog and Oct4 are silenced through increased DNA methylation.

Analysis of the Rassf1A promoter for consensus binding sites (TRANSFAC), reveals direct evidence (ChIP, ENCODE) only for binding by the differentiation-promoting GATA family member $^{36}$, GATA1 (Fig. 1f). GATA transcription factors regulate 
a

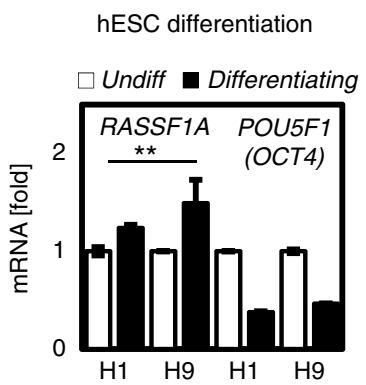

d

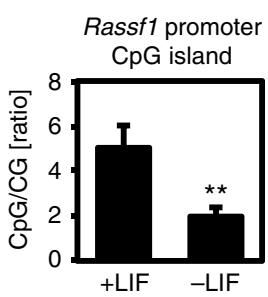

b

mESC differentiation

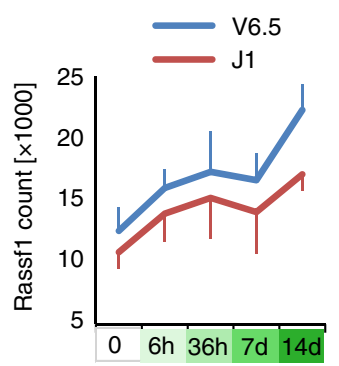

e

H3K4Me3 ChIP

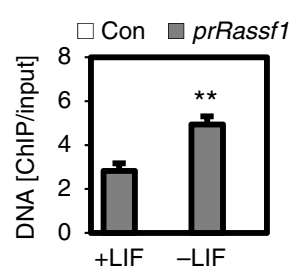

g

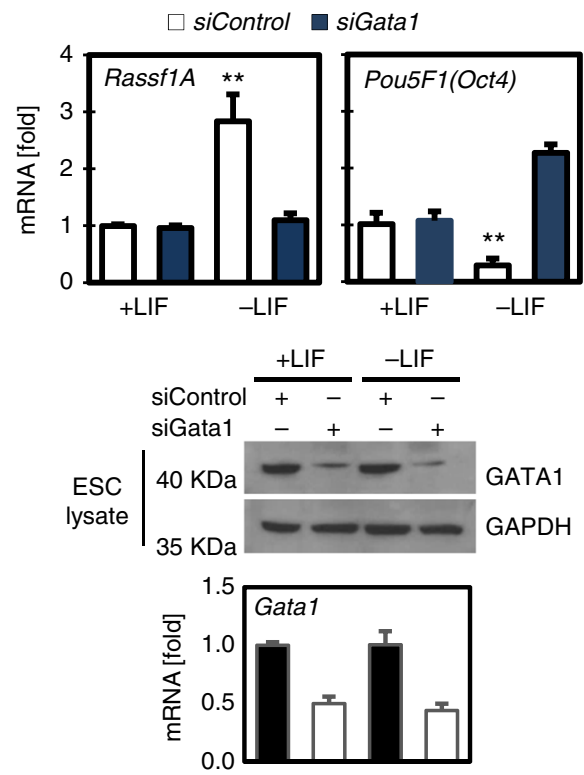

C

+LIF
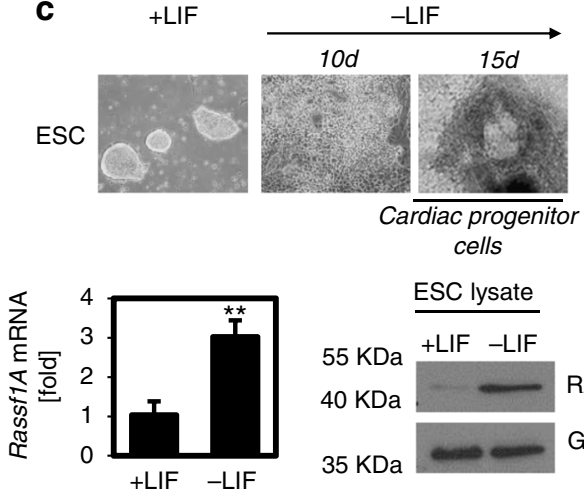

$55 \mathrm{KDa}+$ ESC lysate
$40 \mathrm{KDa}$
$35 \mathrm{KDa}$

RASSF1A

GAPDH

\section{f}

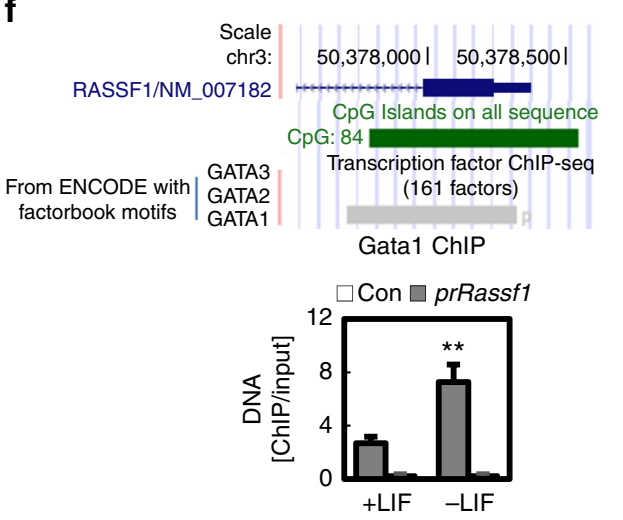

h

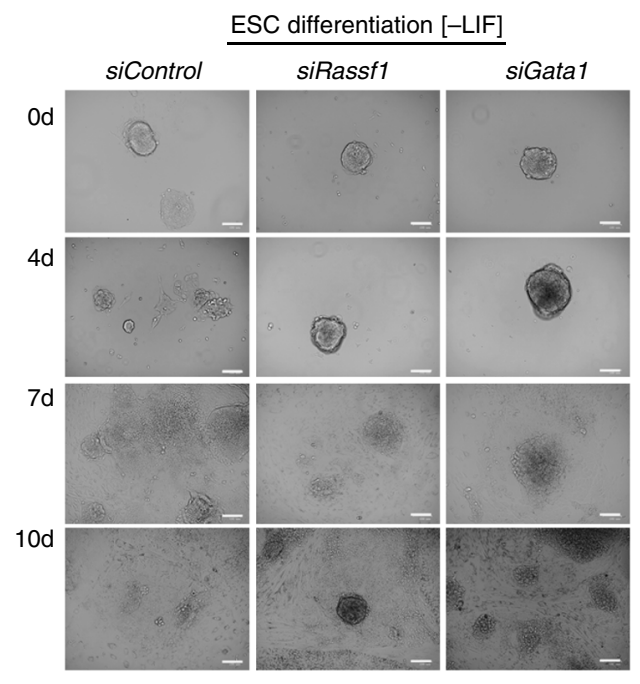

Fig. 1 Epigenetic activation of Rassf1A drives ESC differentiation via GATA1. a RASSF1A and Pou5f1/Oct4 mRNA levels in undifferentiated and differentiating human ESC lines $\mathrm{H} 1$ and H9. Data obtained from published GSE54186 data set. b Rassf1A mRNA levels increase upon LIF withdrawal-mediated differentiation of mouse ESC (mESC) lines V6.5 and J1. Data obtained from published GSE3749 and GDS2672 data sets. c Rassf1A mRNA (bars) and protein (blots) levels from undifferentiated (+LIF) versus differentiated (-LIF) E14Tg2a mESC. See also Supplementary Fig. 1b and Supplementary Movie 1. d Rassf1A CpG island promoter methylation (assessed by qPCR for methylated/unmethylated DNA ratio) and e H3K4me3 levels in pluripotent (+LIF) versus differentiated (-LIF) mESC. See also Supplementary Fig. 1a. f ENOCDE transcription factor ChIP data showing specific binding of GATA1 on the Rassf1A promoter of differentiated hESC and GATA1 ChIP on the Rassf1A promoter of pluripotent (+LIF) versus differentiated (-LIF) ESC. $\mathbf{g}$ qPCR for Rassf1A and Oct4 mRNA levels in response to siRNA-mediated Gatal silencing in the presence or absence of LIF in ESC. Western blotting and qPCR indicate the level of Gata1 KD. See also Supplementary Figs 1d-e, 2c and 5d. h Representative ESC colonies from indicated conditions subject to LIF withdrawal assay. Scale bars: $25-50 \mu \mathrm{m} .{ }^{\star \star} P<0.01$ of Student's $t$-test. Error bars indicate s.e.m. Data shown are representative of at least three independent experiments 
haematopoietic lineage commitment and have been increasingly described to have roles in cell differentiation in intestinal crypts and during early embryogenesis ${ }^{37,38}$. We found selective binding of GATA1 to the Rassf1A promoter and a positive association with Rassf1A transcription in differentiating cells (Fig. If and Supplementary Fig. 1c). Gatal silencing was sufficient to minimise Rassf1A expression in the absence of LIF, while ectopic expression of GATA1 induced expression despite the presence of LIF (Fig. 1g, Supplementary Fig. 1d). Furthermore, reduction of Gata1 mRNA resulted in maintenance of Oct4 expression in the absence of LIF, indicating that ESCs fail to differentiate in the absence of GATA1-driven RASSF1A expression (Fig. 1g, Supplementary Fig. 1d). Taken together, these data indicate that epigenetic changes on the Rassf1A promoter are permissive for GATA1 binding which in turn supports Rassf1A expression upon differentiation (Supplementary Fig. 1e). Loss of either Gatal or Rassf1A expression resulted in ESC colonies failing to appropriately differentiate and persisting as pluripotent-like colonies in the absence of LIF (Fig. 1h).

RASSF1A regulates expression of differentiation genes. To test whether increased levels of RASSF1A plays an active role in differentiation, we first transiently expressed RASSF1A in mESC maintained under pluripotent conditions. RASSF1Apositive cells were less positive for the stem cell marker NANOG (Fig. 2a and Supplementary Fig. 2a, c, e), had reduced number of ESC colonies and reduced expression of the pluripotency gene cassette (Nanog, Oct4, Sox2) (Supplementary Fig. 2b, d). Conversely, ESC depleted of Rassf1A, had enhanced levels of NANOG expression which could be rescued by ectopic expression of zsRASSF1A, but not by a derivative incapable of activating MST kinases due to deletion of the C-terminus (Fig. $2 \mathrm{~b}$ and $\mathrm{c}$ and Supplementary Fig. 2c). Additionally, ESC depleted of Rassf1A displaying increased Nanog, Oct4, Sox2 demonstrated a reciprocal decrease in the expression of genes involved in lineage commitment and had increased pluripotentlike ESC colonies (Fig. 2d and Supplementary Fig. d, f). To determine whether ESC potency was directly regulated by RASSF1A, we performed RNA sequencing in shRassf1Aexpressing ESC versus control (shGFP) (Fig. 2e). In the absence of RASSF1A, genes required to maintain pluripotency and selfrenewal were upregulated, including components of the TGF $\beta$, WNT and Hippo pathways, SMAD2/3, LEF1 and YAP targets, whereas factors promoting ESC differentiation, such as $\mathrm{p} 53 / \mathrm{p} 73$ targets, Cdkn1A (p21) and Gata4, were downregulated (Fig. 2e and Supplementary Data 1). Expression of RASSF1A is therefore sufficient to directly regulate expression of pluripotency versus differentiation genes.

A quaternary YAP complex induces Oct4 expression. RASSF1A is an established component of the Hippo pathway; we therefore set out to investigate whether its activation affects YAP-TEAD and YAP- $\beta$-catenin transcriptional complex formation/activity, which both have a pivotal role in maintaining stem cell selfrenewal $^{2,5,13}$. YAP ChIP-seq demonstrated reduced association (occupancy shift) of YAP on target genes in ESC expressing RASSF1A compared to controls. Previously documented YAP-TEAD $/ \beta$-catenin target genes appeared more sensitive to RASSF1A levels than classical YAP-TEAD targets which displayed equivalent peaks in RASSF1A-expressing ESC and controls (Fig. 3a and Supplementary Fig. 3b). To assess the transcriptional activity of YAP in response to RASSF1A expression, we combined genome-wide profiling of YAP binding (ChIPseq) with transcriptome analysis (RNAseq) in shGFP and shRassf1A-expressing ESC lines. There was a substantial enrichment of YAP binding events in the immediate vicinity of transcription start sites of genes differentially expressed following knockdown of Rassf1A, indicating the major transcriptional activity of YAP is negatively regulated by RASSF1A in ESC (Supplementary Fig. 3a). As expected, TEAD was the most upregulated transcription factor motif at sites proximal to YAP targets in the absence of RASSF1A (Fig. 3b). Additionally, motifs of several pluripotency-related transcription factors including TCF7L1/2(TCF3/4), ESRRB, and SMAD2/3 ${ }^{39}$ (Fig. 3b and Supplementary Data 2) were increased in the absence of RASSF1A. TEAD2, TCF3/4 and $\beta$-catenin activate Oct4 transcription via direct binding of its distal enhancer ${ }^{2}, 40$. We therefore reasoned that RASSF1A may redirect YAP from active complexes on the Oct4 promoter, preventing expression. Indeed, direct ChIP-qPCR for YAP, TEAD2 and $\beta$-catenin demonstrated that in the presence of RASSF1A; YAP, TEAD2 and $\beta$-catenin have decreased affinity for the Oct4 promoter (Fig. 3c). In keeping with our previous results, analysis of the YAP interactome in ESCs revealed that TEAD2, TCF3 and its co-factor $\beta$-catenin ${ }^{41}$ lose association with YAP in response to RASSF1A expression (Fig. 3d, Supplementary Fig. 3c and Data 3). We next examined our separation of ESC lysates on gel filtration column and observed co-purification of total YAP in the same fraction as $\beta$ catenin, TCF3 and TEAD2 in the presence of LIF, however, the co-purification is lost upon differentiation (-LIF day 4; Fig. $3 e$ and Supplementary Fig. 3d).

To confirm these observations, we depleted Rassf1A from ESCs either transiently using two independent siRNA sequences or stably, using lentiviral vectors and determined interaction by co-immunoprecipitation. Loss of Rassf1A resulted in elevated levels of $\beta$-catenin and TCF3 in YAP and TEAD2 immunoprecipitates (Fig. 3f, Supplementary Fig. 3e, f, g and 4a). The identification of TCF3 in YAP IPs suggests the existence of a YAP-TEAD2- $\beta$-catenin trimeric complex, which may include the canonical $\beta$-catenin partners TCF3/4. To address this, we employed stable shGFP, shRassf1A, shTead2 and shRassf1A/ shTead2 ESC lines transfected with siRNAs to either $\beta$-catenin, TCF3 and TCF4, or non-targeting controls (siNT) (Supplementary Fig. $4 \mathrm{~b}$ and $\mathrm{c}$ ) and performed YAP ChIP using primers identifying either TEAD or $\beta$-catenin/TCF binding sites on the Oct4 promoter. Knockdown of shRassf1A led to an increase in YAP occupancy of both sites on the Oct4 promoter which was dependent on $\beta$-catenin, TCF3 and TCF4 (Fig. 3g). This implies that $\beta$-catenin localisation to the Oct4 promoter is TEAD2dependent and suggests that the YAP-TEAD2- $\beta$-catenin-TCF3 complex acts as transcription-promoting unit on Oct4. In support of this, silencing of $\beta$-catenin, TCF3 or TCF4 together with RASSF1A (shRassf1A/sißcat; shRassf1A/siTcf7l1; shRassf1A/ siTcf7l2), reduced TEAD2 promoter binding (Fig. 3g). YAP depletion disrupted the complex formation, confirming that RASSF1A-mediated regulation of TEAD2- $\beta$-catenin was indeed YAP-dependent and responsible for Oct4/Sox2/Nanog expression and pluripotency (Supplementary Fig. 3g). Moreover, we performed TEAD2 and $\beta$-catenin ChIP on the same target sites of the Oct4 promoter and obtained similar results (Supplementary Fig. 5a and $\mathrm{b}$ ). In line with the transcriptional regulation of Rassf1A, GATA1 overexpression also suppresses the ability of YAP to ChIP at both binding sites in the Oct4 enhancer (Supplementary Fig. 5c). Intriguingly, in addition to silencing of YAP, TEAD2 or $\beta$-catenin (Supplementary Fig. $4 \mathrm{~d}-\mathrm{f}$ ), silencing of MST2-LATS1 in the absence of RASSF1A ablated Oct4 and Sox2 upregulation (Supplementary Fig. 5d). This suggests that the RASSF1A signalling switch of the YAP partner not only operates through the Hippo cassette, but may be required to facilitate YAP- $\beta$-catenin transcription over YAP-TEAD alone as suggested previously ${ }^{26}$. 
RASSF1A promotes YAP and p73 binding to differentiation genes. LATS-dependent phosphorylation of YAP regulates its activity in response to various signals ${ }^{42,43}$. As shown previously, the key LATS-dependent regulatory site, pS127-YAP, is undetectable in pluripotent ESCs and increases during ESC differentiation ${ }^{5}$. We also find this is the case, however, rather than purely cytoplasmic a proportion of pS127-YAP remains nuclear at day 5 -LIF and becomes largely nuclear by day 15 (Fig. 4a). We also reasoned that since RASSF1A drives the formation of proapoptotic YAP-p73 complexes in cancer ${ }^{21,23,24}$, expression of RASSF1A may also stabilise YAP-p73 complexes in ESCs and promote differentiation. We next examined our Gel filtration separation of ESC d4 -LIF lysates and observed co-purification of a small pool of total YAP marked with pS127-YAP in the same high molecular weight fraction as p73 (Fig. 4b), indicating the potential for complex formation. We performed a a

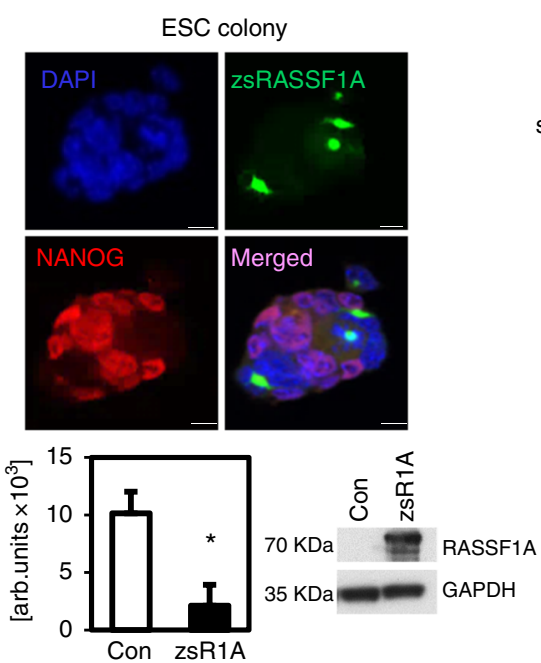

C

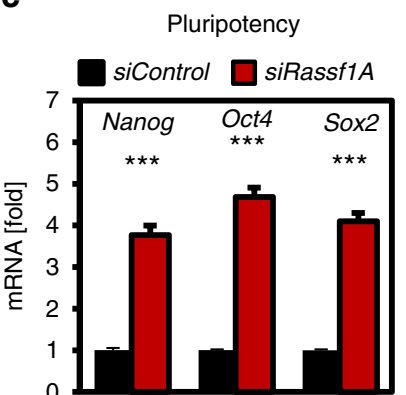

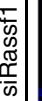

b
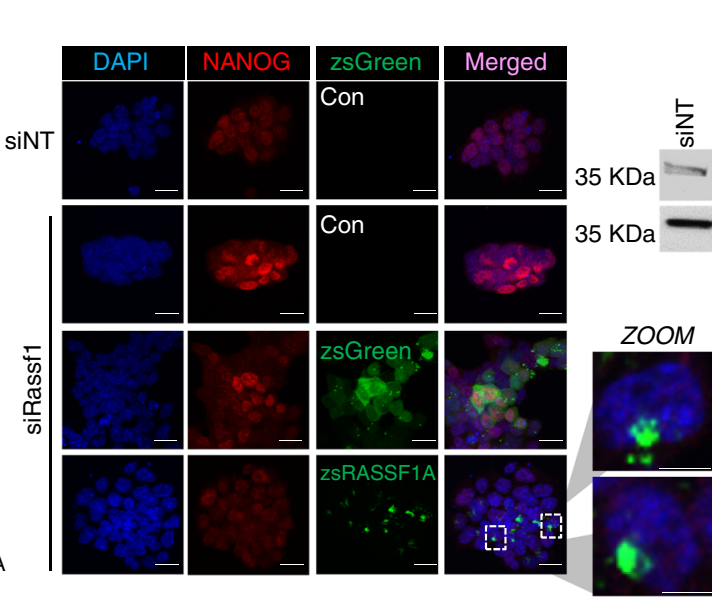

ESC Lysate
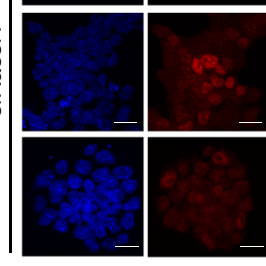

Li:

(b)

d

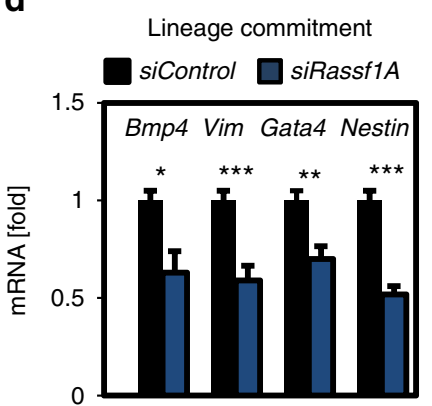

e

ESC shRassf1:con

RNASeq

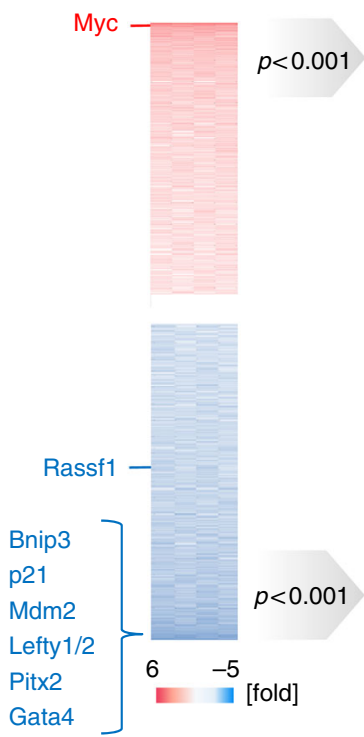

\begin{tabular}{|c|c|c|c|}
\cline { 2 - 4 } RNASeq \\
\begin{tabular}{|c|c|c|c|}
\hline Gene sets [UP n=278] & p-value & FDR q-value & $\begin{array}{c}\text { Predicted } \\
\text { significance }\end{array}$ \\
\hline ESC (V6.5 cell)_Pluripotency & $3.81^{\mathrm{E}-16}$ & $2.37^{\mathrm{E}-13}$ & Embryoid bodies \\
ESC (J1 cell)_Pluripotency & $2.67^{\mathrm{E}-15}$ & $1.21^{\mathrm{E}-12}$ & Embryoid bodies \\
SMAD2/3 targets & $2.06^{\mathrm{E}-14}$ & $7.88^{\mathrm{E}-12}$ & TGF $\beta$ \\
LEF1_up & $1.27^{\mathrm{E}-03}$ & $9.55^{\mathrm{E}-03}$ & WNT \\
YAP_Cordenonsi & $5.04^{\mathrm{E}-03}$ & $2.41^{\mathrm{E}-02}$ & Hippo \\
\hline
\end{tabular}
\end{tabular}

\begin{tabular}{|c|c|c|c|}
\hline Gene sets [DOWN n=353] & $\mathrm{p}$-value & FDR q-value & $\begin{array}{c}\text { Predicted } \\
\text { significance }\end{array}$ \\
\hline ESC(V6.5 cell)_differentiation & $1.77^{\mathrm{E}-27}$ & $3.40^{\mathrm{E}-24}$ & EBb differentiation \\
\hline HYPOXIA_ & $4.60^{\mathrm{E}-27}$ & $6.65^{\mathrm{E}-24}$ & Hif1a activation \\
\hline MDM4_KO & $2.85^{\mathrm{E}-25}$ & $2.35^{\mathrm{E}-22}$ & p53/p73 activation \\
\hline ESC ( $\mathrm{J} 1$ cell)_differentiation & $1.12^{\mathrm{E}-24}$ & $8.06^{\mathrm{E}-22}$ & EB differentiation \\
\hline Decitabine induced genes & $4.91^{\mathrm{E}-23}$ & $2.36^{\mathrm{E}-20}$ & $\begin{array}{l}\text { RASSF1A } \\
\text { expression }\end{array}$ \\
\hline
\end{tabular}


phosphoproteomic analysis of FLAG-YAP immunoprecipitates from ESCs in the presence or absence of RASSF1A and, in line with a role for the Hippo pathway, S127-YAP was phosphorylated when RASSF1A was expressed (Fig. 4c, Supplementary Fig. 6a and Data 4). Moreover, in pluripotent ESCs, YAP-TEAD$\beta$-catenin-TCF3 complexes were readily detected, but were reduced in favour of YAP-p73 in the presence of RASSF1A (Fig. 3e, f and Supplementary Data 3). To investigate whether YAP-p73 complexes play a role in ESC fate determination as described for $\mathrm{p} 73^{15}$, we examined genome-wide YAP DNA binding peaks (ChIPseq). This showed that RASSF1A enriched YAP binding to several differentiation gene promoters (e.g., $p 53$, Fbxw7, Klf3) containing p73 binding consensus motifs 44,45 (Supplementary Data 5). p53/p73 can promote BMP4 expression during differentiation ${ }^{46}$ and Fbxw7 is an E3 ligase that targets cMYC, which is linked with development of endothelial and hematopoietic lineages in the embryo ${ }^{47}$ and regulates GATA $4^{48}$.

YAP and p73 complex stabilisation by RASSF1A is dependent on pS127. As RASSF1A signaling stabilises YAP-p73 complexes (Fig. 4d), we speculated that the formation of these complexes may be dependent on phosphorylation of S127. To directly address this, ESCs were transiently transfected with either FLAG-YAP or a non-phosphorylatable ${ }^{3}$ FLAG-YAP-S127A mutant and complexes were retrieved by co-immunoprecipitation of the FLAG epitope. RASSF1A expression promoted the switch of YAP association with TEAD2 and $\beta$-catenin to p73, however, in the context of the S127A mutant TEAD2 and $\beta$-catenin binding was maintained (Fig. 4e). Furthermore, we performed a proximity ligation assay (PLA), which provided evidence for close association of pS127-YAP with p73 and, moreover, revealed that pS127-YAP-p73 complexes could only be detected in RASSF1Aexpressing cells (Fig. 4f and Supplementary Fig. 6b). ChIP experiments using S127A-YAP and sip73 indicated that binding of YAP to differentiation gene promoters is pS127-YAP and p73 dependent (Fig. $4 \mathrm{~g}$ and Supplementary Fig. $6 \mathrm{c}$ and $\mathrm{d}$ ). Taken together, these observations imply that pS127-YAP contributes to ESC self-renewal and differentiation in response to RASSF1A levels. In line with this, RASSF1A overexpression was accompanied by increased expression of 'first wave' mesoderm, endoderm and ectoderm-specific differentiation markers in a p73dependent manner ${ }^{15}, 49$ (Fig. $4 \mathrm{~h}$ and Supplementary Fig. 6e). We also confirmed that normal differentiating ESC require RASSF1A expression for YAP to optimally bind to Klf3, Fbxw7 and p53 promoters and promote transcription (Supplementary Fig. 6f, g). Finally, to ultimately test our model, ESC expressing RASSF1A or control plasmid were induced to differentiate towards the neural lineage by incubating in N2B27 media containing RA. RASSF1Aexpressing ESC expressed higher levels of the neuronal markers PAX3 and SOX1, in a p73-dependent manner (Fig. 4i). This indicates the requirement for an intact RASSF1A-YAP-p73 axis during ESC differentiation.
RASSF1A regulates early embryonic differentiation in vivo. In support of our model, expression profiles indicate that targeted inactivation of OCT4 in ESCs, pushing cells out of pluripotency towards differentiation, also increases expression of GATA1, RASSF1A and p53/p73 targets (e.g., Mdm2, Cdkn1A, Fbxw7) (Fig. 5a). In the early embryo, epigenetic control of totipotency is governed by Brgl, and when Brgl is depleted in two-cell embryos, RASSF1A expression is increased along with associated differentiation genes (Fig. 5a and Supplementary Fig 7a). As YAP drives the early specification of cell fate in embryos, we reasoned that RASSF1A may play a role much earlier in development than previously anticipated. During early development the expression pattern of Oct4 is reciprocal to Rassf1A indicating that our observations in cultured mESCs may be directly relevant in vivo ${ }^{50,51}$ (Fig. 5b). Nuclear localisation of YAP during 2-8 cell stages of the embryo (Fig. 5c), when Rassf1A expression is low, is coincident with Oct4 transcription, which in turn triggers the expression of additional core stem-cell markers (NANOG, SOX2) in the $\mathrm{ICM}^{52}$. To functionally test if reciprocal expression of OCT4 and RASSF1A regulates the switch to differentiation in the embryo, mouse pre-implantation embryos were microinjected with either non-targeting siRNA (siNT), siRassf1A, mRNA encoding green florescent protein (Ctrl GFP or zsGreen) or GFPtagged Rassf1A mRNA (zsRassf1A) at the zygote stage and allowed to develop to blastocysts (Supplementary Fig. 7b). Consistently, at all developmental stages examined (2-cell, 4-cell, 8cell, 16-cell and 32-cell stage), RASSF1A loss led to maintenance of pluripotency markers (Fig. 5d), whereas RASSF1A induction drastically reversed the phenotype (Fig. 5e), suggesting that RASSF1A can actively regulate the core pluripotency network in ESCs and the mouse embryo. Embryos injected with Rassf $1 \mathrm{~A}$ mRNA concentrations above $200 \mathrm{ng} / \mu \mathrm{L}$ did not develop and concentrations higher than $300 \mathrm{ng} / \mu \mathrm{L}$ led to $100 \%$ lethality ( 0 out of 15 embryos survived) (Fig. 5f and Supplementary Fig. 7d). Given the positive correlations between stem cell marker depletion and embryonic lethality ${ }^{53}$, we reasoned that the induced lethality was due to premature RASSF1A-driven embryonic differentiation. As RASSF1A appeared to exert its fatedetermining functions through p73 in ESCs, we postulated that silencing of p73 should reverse the effects seen upon RASSF1A induction in the mouse embryo. To test this hypothesis, we injected embryos with $1000 \mathrm{ng} / \mu \mathrm{L}$ of control or Rassf1A mRNA, a concentration which exceeded the lethality threshold, together with siRNA to p73. Control embryos were able to form blastocysts (10 out of 15), while RASSF1A-expressing embryos arrested and died ( 0 out of 15 survived; Fig. $5 \mathrm{~g}$ ). Strikingly, the lethal phenotype was rescued in embryos microinjected with both Rassf1A mRNA and sip73, (11 out of 15 embryos survived; Fig. 5g). Knockdown of p73 alone is not accompanied by early developmental defects and embryos are viable at least up to the blastocyst stage (Supplementary Fig. 7e). Together, these data indicate that the key effector of RASSF1A signaling in development is indeed p73.

\footnotetext{
Fig. 2 RASSF1A regulates the ESC core pluripotency network. a Representative fluorescent images of Nanog and zsRASSF1A (zsR1A)-expressing cells in mouse ESC colonies. Bar graph and western blotting represent quantification of Nanog and RASSF1A levels, respectively. $\mathbf{b}$ Nanog immunofluorescence in siNT versus siRASSF1A-transfected ESC. Additional expression of siRASSF1A-resistant zSRASSF1A rescues the phenotype in siRASSF1A-transfected ESC, quantified in the displayed bar graphs and additionally demonstrated by Western blotting. Zoom in displays RASSF1A localisation peripherally to the nucleus at the microtubule organising centre, in zsRASSF1A-expressing cells. Validation using a second siRNA to Rassf1A is provided in Supplementary Fig. 2g. c qPCR for core stem cell markers from ESC in b. Ectopic expression of RASSF1A reverses the ESC pluripotent phenotype, see Supplementary Fig 2a-e. d qPCR for germ layer-specific differentiation markers in ESC subject to LIF withdrawal. e RNAseq analysis in shRNA-expressing ESC versus control (shGFP) reveals establishment of self-renewal and pluripotency signatures in the absence of Rassf1A, Supplementary Data 1. Scale bars: 25 and 50 $\mu \mathrm{m} .{ }^{\star} P<0.05,{ }^{\star \star} P<0.01$ and ${ }^{\star \star \star} P<0.001$, respectively, of Student's $t$-test. Error bars indicate s.e.m. Data shown are representative of at least three independent experiments
} 
RASSF1A represents a barrier to reprogramming in iPSC. Rass $f 1 A^{-1-}$ mice are viable, potentially due to compensation from the close homologue Rassf5 (49\% identity) which has similar effects in the early embryo (Supplementary Fig 7c), but tumour prone. To determine whether tumour formation was due to a failure to control the YAP pluripotency to differentiation switch we isolated mouse embryonic fibroblasts (MEFs) from Rassf1A $-/-$ and Rassf $1 A^{+/+}$littermates and subjected them to somatic cell reprogramming as previously described ${ }^{54,55}$ (Fig. 6a and Supplementary Fig. 8a). Colony morphology, NANOG and alkaline phosphatase staining indicated higher efficiency of iPSC genera-

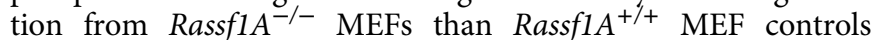
(Fig. 6b), suggesting that RASSF1A presents a barrier to reprogramming in agreement with results in ESCs. To confirm that the enhanced reprogramming efficiency was due to RASSF1A, we retrovirally introduced RASSF1A into Rassf1A ${ }^{-/-}$MEFs (Rassf1A a

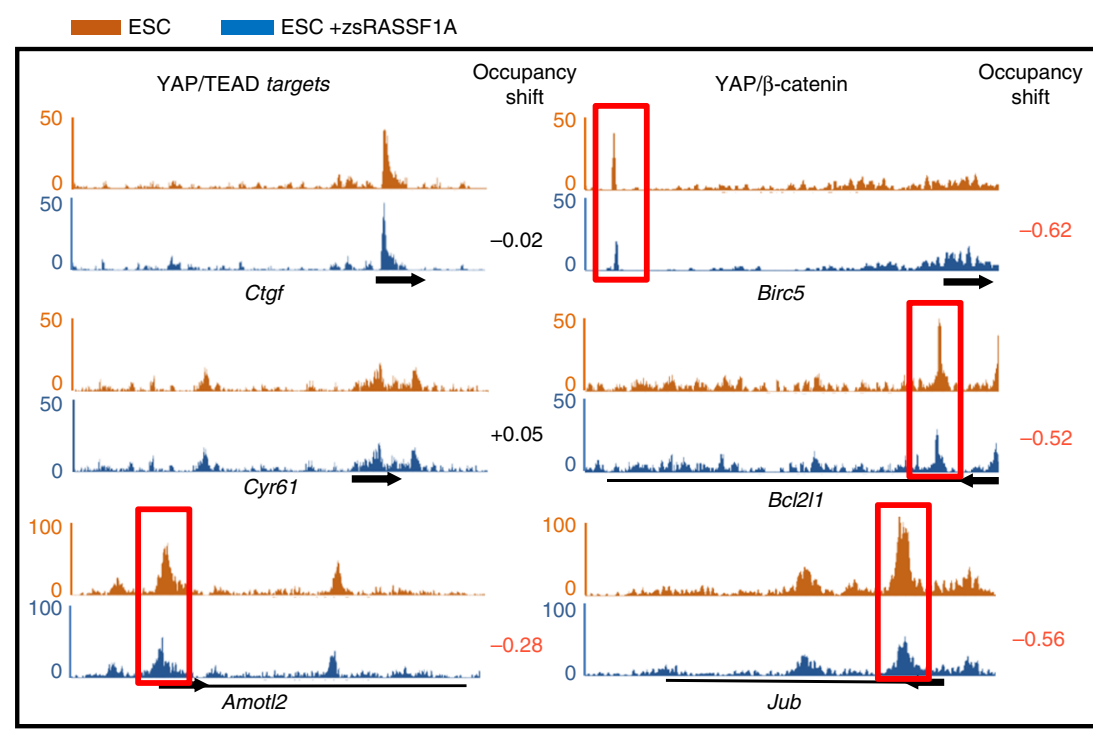

b

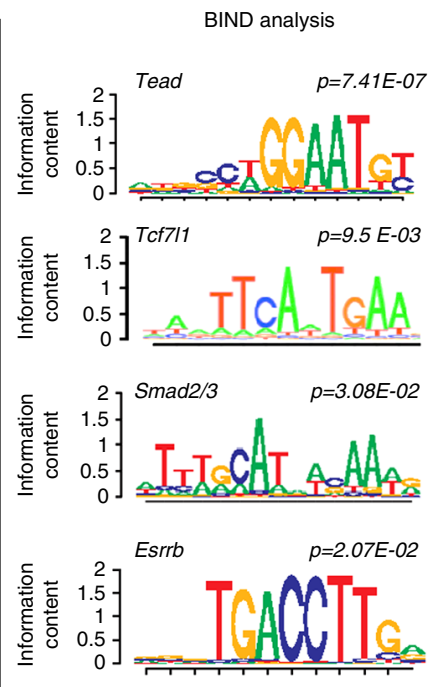

C

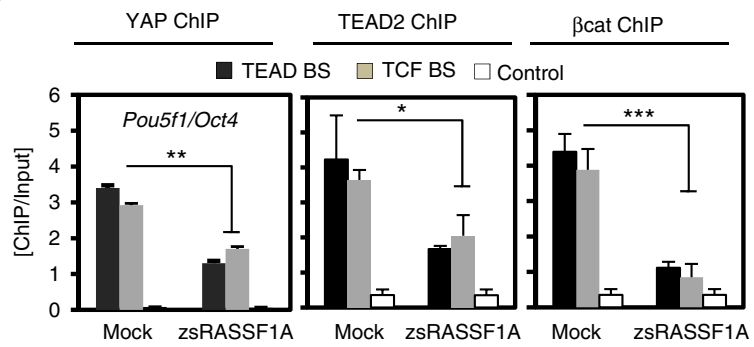

d

YAP IP LC MS/MS

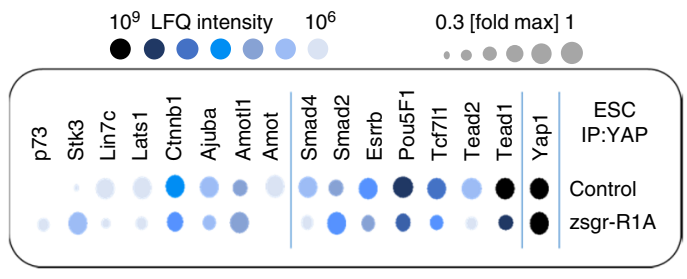

e

S Size exclusion chromatography

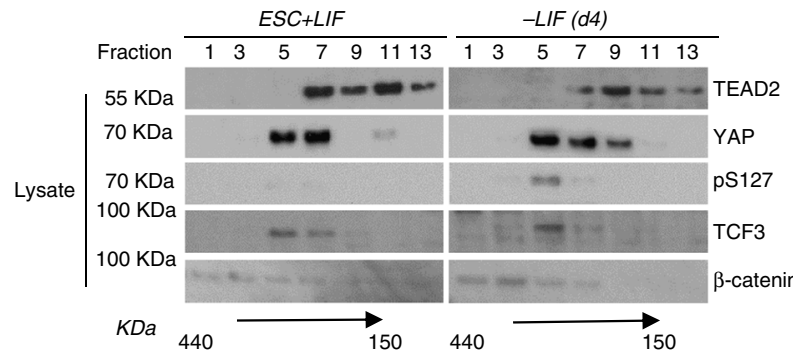

f

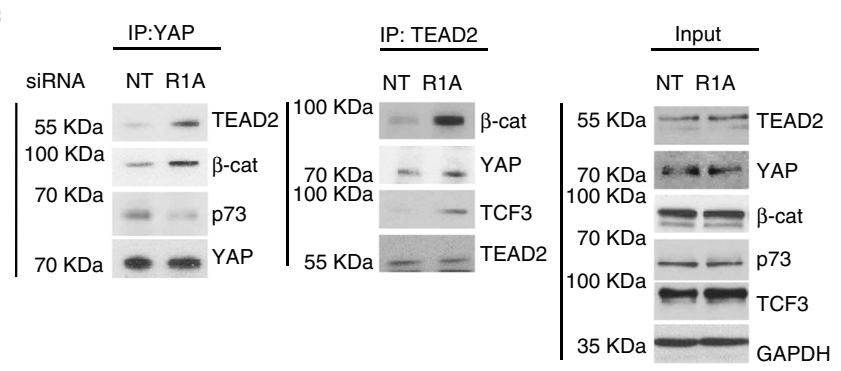

g

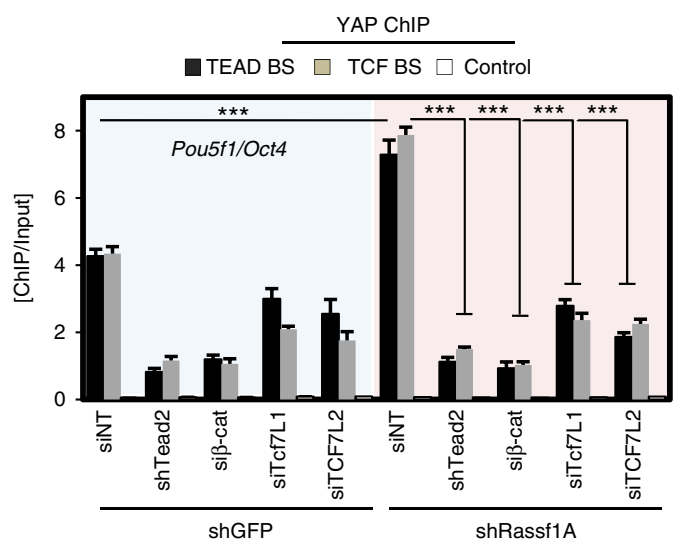


$-/-R A S S F 1 A)$ and found that iPSC efficiency was reduced to levels similar to Rassf1 $1 A^{+/+}$MEFs (Fig. 6b).

We observed that in Rassf1A $A^{-/-}$iPSCs, endogenous mRNA and protein levels of the core stem cell markers were elevated in comparison to Rassf $1 A^{+/+}$or Rassf1 $1 A^{-/-R A S S F 1 A}$ iPSC (Fig. 6c; Supplementary Fig. 8c). This indicates that despite equivalent expression of the reprogramming vector (Supplementary Fig. 8b), iPSC colonies were restricted from commitment to pluripotency by RASSF1A. We next wanted to confirm the regulatory switch in transcription factor association was apparent in iPSC and found reduced YAP association with p73 in Rassf $1 A^{-/-}$iPSCs compared to control or Rassf1 $1 A^{-/-R A S S F I A}$ iPSC and reciprocally increased association with TEAD2 and $\beta$-catenin (Supplementary Fig. $8 \mathrm{~d}$ ).

In agreement with our findings in ESCs, a proteomics screen in iPSCs identified enriched association of YAP with pluripotency associated factors in Rassf $1 A^{-/-}$iPSCs compared to Rassf $1 A^{+/+}$ or Rassf1A $1 A^{-/-R A S S F 1 A}$ iPSCs (Fig. 6d and Supplementary Data 6). pS127-YAP levels were elevated and positively associated with differentiation, promoting transcription factors in Rassf $1 A^{+/+}$or

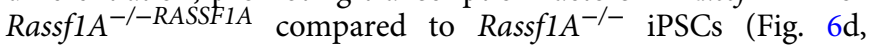
Supplementary Fig. 8e and Supplementary Data 4), thus indicating that RASSF1A is a barrier in reprogramming to pluripotency. Furthermore, following induction of differentiation via LIF withdrawal, we observed the expected increases in meso-endodermal and ectodermal markers in Rassf $1 A^{+/+}$and Rassf $1 A^{-/-R A S S F 1 A}$ iPSC, whereas these were lower in Rassf $1 A^{-/-}$ iPSC indicating a reduced capability to enter a differentiation programme (Supplementary Fig. $8 \mathrm{f}$ and g). LIF withdrawal assays demonstrated that the majority $(\sim 80 \%)$ of $R a s s f 1 A^{+/+}$and Rassf $1 A^{-/-R A S S F 1 A}$ colonies began to collapse at 4 days (80 and $95 \%$, respectively), while $45 \%$ of Rassf $1 A^{-1-}$ colonies sustained their round shape (Fig. 6e and Supplementary Fig. 8h), suggesting delayed differentiation in keeping with higher levels of core pluripotency markers.

To assess differentiation capacity, iPSCs derived from round colonies were challenged to form embryoid bodies (EBs) and again differentiate towards the neural cell lineage, using defined media containing N2B27 and RA. Neural progenitor marker expression demonstrated that Rassf1 $1 A^{-/-}$iPSCs were less efficient at generating neural progenitors (Fig. 6f), indicating a requirement for RASSF1A to promote differentiation. To further validate these results we employed a second iPSC differentiation protocol of EBs towards the dendritic cell (DC) lineage, following previously established methods ${ }^{56}$. FACS staining for expression of the DC-specific cell surface marker cd54 demonstrated that $R a s s f 1 A^{+/+}$iPSC were more efficient at differentiating to DCs compared to Rassf1 $1 A^{-/-}$iPSC, whereas both were equally responsive to LPS-mediated stimulation indicating that overall capacity to differentiate was not affected (Supplementary Fig. 8i). Taken together, these data suggest that iPSCs lacking RASSF1A exhibit general resistance to differentiation signals and maintain proliferation, illustrated by insensitivity to
TGF- $\beta$-mediated inhibition and elevated Ki67 staining (Supplementary Fig. 8j and k).

\section{Discussion}

The Hippo pathway is established to restrict pluripotency by preventing YAP-TEAD-mediated activation of Oct4 transcription $^{52}$. WNT/ $\beta$-catenin signalling drives maintenance of pluripotency by switching the WNT effector TCF3 from a repressor to an activator of Oct4 transcription in ESCs ${ }^{57}$. Here we demonstrate that these two events are mutually dependant and that both YAP-TEAD and $\beta$-catenin-TCF3 cooperate to promote transcription of Oct4 from its distal enhancer to sustain pluripotency in ESCs. Upon differentiation of ESCs, we find that the differentiation factor GATA1 is expressed much earlier than previously appreciated and enhances expression of the MST1/2 kinase scaffold RASSF1A ${ }^{37,38}$. RASSF1A drives Hippo pathway activation and pS127-YAP, which reduces YAP-TEAD interaction and transcription of Oct4. Of note, MST1/2 kinases are regulated by WW45/Sav1 in addition to RASSF1A, however WW45/Sav1 levels were below detection in human and mouse ESC. We find that RASSF1A-mediated Hippo activation not only restricts YAP-TEAD binding to Oct4, but actively promotes YAP binding to $\mathrm{p} 73$ to active transcriptional complexes required for ESC differentiation. Our data suggest that a TEAD-defective mutant of YAP may remain capable of supporting a p73mediated event but this remains to be tested. Moreover, we provide evidence that the transcriptional switch is important in vivo and responsible for the recently described role for p73 in early lineage specification.

Alteration of DNA methylation is widely appreciated as a key regulatory event upon fertilisation and during induction of pluripotency in differentiated cells ${ }^{58}$. Under both processes, erasing epigenetic memory is key in allowing transition to distinct cellular fates. Here we find that the DNA demethylation in ESCs and pre-implantation embryos leads to reversion of the poised state of the Rassf1A promoter and leads to activation of Rassf $1 A$ transcript expression towards blastocyst formation. This is a developmentally conserved event with evidence for Rassf $1 \mathrm{~A}$ demethylation during zebrafish, mouse and human embryogenesis $^{31,59}$. In humans, RASSF1A expression has been described as one of the main clinical biomarkers of placental defects in pregnancies ${ }^{33,60}$. Similarly, defects in p73 also associate with risk of recurrent pregnancy loss ${ }^{61}$, and our data would suggest that this may be linked to Hippo pathway signalling.

The Hippo pathway also plays a major role in the preimplantation embryo, where YAP is required for specification of the TE and $\mathrm{ICM}^{4,32}$. However, Yap ${ }^{-/-}$mice do not arrest until developmental stage E8.5, implying the genetic model does not recapitulate the early developmental role ${ }^{62}$. Despite a crucial role in differentiation and lineage commitment, $t p 73^{-/-}$mice only develop with late developmental defects due to compensation

Fig. 3 A quaternary YAP-TEAD2/ $\beta$-catenin-TCF complex is essential for maintaining expression of Oct4. a YAP ChIP-seq in zsRASSF1A-overexpressing versus control ESC depicting reduced occupancy of YAP-TEAD and YAP- $\beta$-catenin target genes in the presence of RASSF1A. See also Supplementary Fig. 3a, b, 4 and Data 6. b Enriched sequence motifs $(P<0.05)$ identified in the proximity of upregulated YAP target genes upon RASSF1A loss in ESC. See also Supplementary Data 2. c YAP, TEAD2 and $\beta$-catenin ChIP on TEAD2 and TCF binding sites (BS) on the ESC Pou5f1/Oct4 promoter in response to indicated conditions. $\mathbf{d}$ Proteomics analysis for YAP binding partners in empty vector versus zsRASSF1A-transfected ESC. Factors modifying their affinity with YAP are represented by relative abundance of peptides (LFQ intensity) and fold difference to maximum. All mass-spec intensities were normalised to YAP intensities. See also Supplementary Fig. 3c and Data 3. e Size exclusion chromatography of ESC lysates via Gel filtration column and western blotting with indicated antibodies. See also Supplementary Fig. 3d. $\mathbf{f}$ Western blotting of YAP and TEAD2 immunoprecipitates in response to transient depletion of RASSF1A. Validation using \#2 siRassf1A and shRassf1A is provided in Supplementary Fig. 3e-g. $\mathbf{g}$ YAP ChIP at TEAD and $\beta$-catenin/TCF binding sites (BS) on the ESC Pou5f1/Oct4 promoter in response to stable ablation of RASSF1A versus control. ${ }^{\star} P<0.05,{ }^{\star \star} P<0.01$ and ${ }^{\star \star \star} P<0.001$, respectively, of Student's $t$-test. Error bars indicate s.e.m. Data shown are representative of at least three independent experiments 
from $\mathrm{p} 53$ in the early embryo ${ }^{15}$. Similarly, individual genetic ablation of Hippo pathway kinases $\mathrm{Ndr1/2}$, Lats1/2 and Mst1/2 only display phenotypes upon dual deletion of both isoforms, and tumour prone Rassf $1 A^{-/-}$mice are viable, reportedly due to compensation from the homologous family member Rassf5 $5^{63}, 64$. In keeping with the widespread role as a tumour suppressor in humans, Rassf $1 A^{-/-}$mice succumb to a variety of tumours, indicating a potential weakening of a pluripotency to differentiation switch that is not completely compensated for in the null mice. This is supported by increased pluripotent reprogramming efficiency of Rassf $1 A^{-/-}$MEFs suggesting that RASSF1A maintains differentiation of fibroblasts and represents a barrier to reprogramming during iPSC generation. Moreover, the loss of RASSF1A in human tumours is a

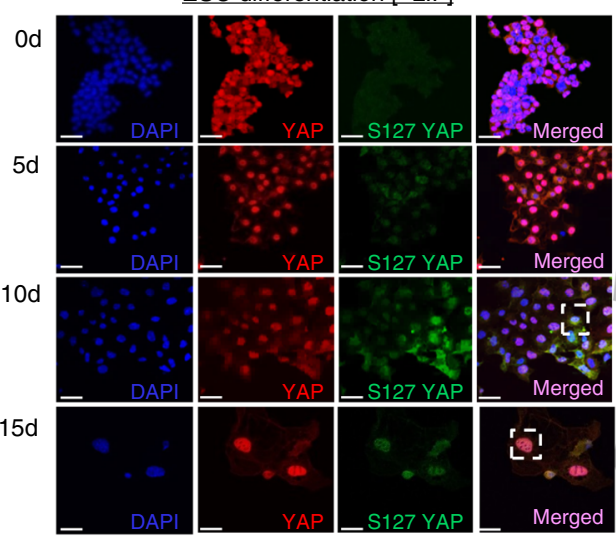

d

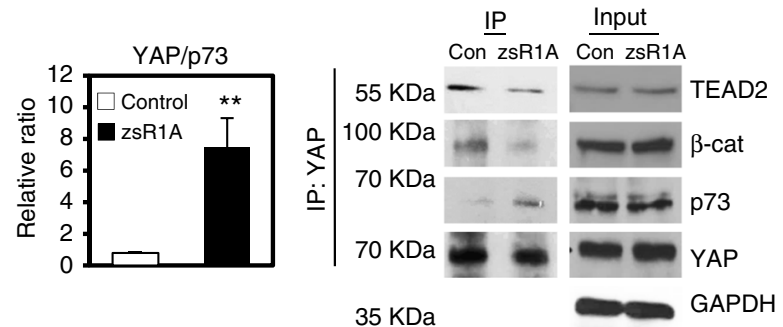

b

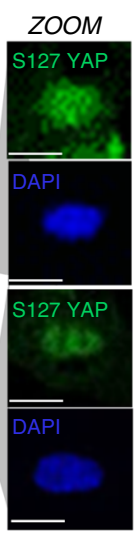

$\mathbf{f}$
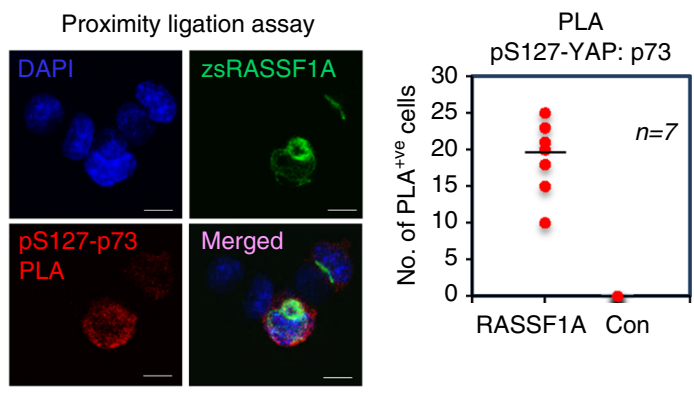

g

Flag-YAP ChIP

- Empty vector $\square$ RASSF1A $\square$ Control

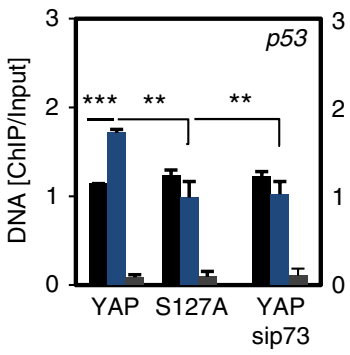

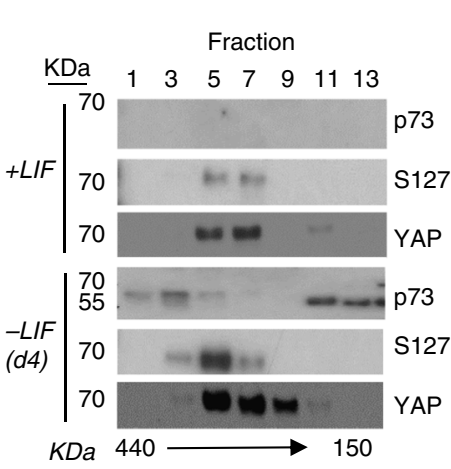

FLAG-YAP $\quad \stackrel{\check{D}}{+} \frac{\mathrm{R} 1 \mathrm{~A}}{+-}+\frac{\mathrm{R} 1 \mathrm{~A}}{+-}$

${ }^{3} \mathrm{FLAG}-\mathrm{S} 127 \mathrm{~A}-\ldots+\ldots$

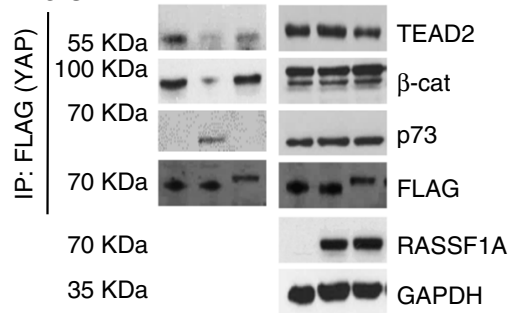

h
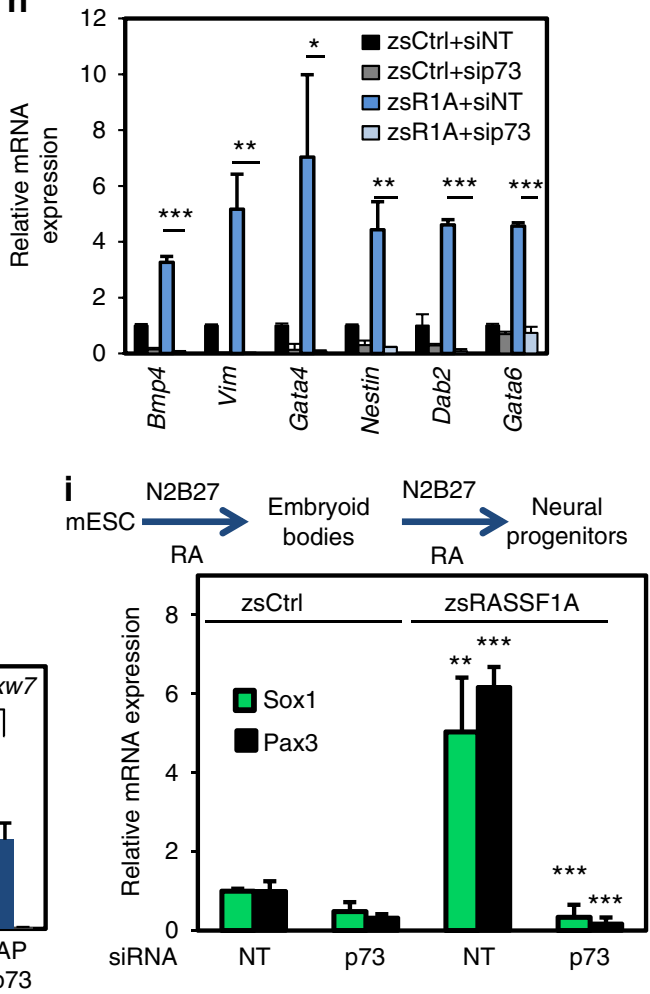
associated with lower differentiation grade and increased stem cell characteristics.

Taken together, our findings clearly show that RASSF1A mediates a switch of factors that YAP promotes transcription through and that this is crucial in maintaining the balance between pluripotency and differentiation. Thus, RASSF1A is a key mechanism that determines transition from pluripotency to differentiation in ESCs via WNT/Hippo pathway transcription factor competition. As RASSF1A is an established tumour suppressor, our finding of a major role in stem cell biology highlights a significant regulatory event that may contribute to tumourigenesis and provides a link between tumour formation and dedifferentiation.

\begin{abstract}
Methods
Cell lines and culture. E14Tg2a mouse ESCs (obtained from ATCC), iPS cells and the subsequently derived stable lines (shGFP, shRassfla, shTead2, shRassfla/ shTead2-expressing cell lines) were maintained in cell culturing medium consisting of DMEM (High Glucose, Sodium Pyruvate, Gibco) supplemented with $0.01 \%$ LIF (ESGRO, Millipore), 10\% Knockout Serum Replacement (Invitrogen), 2\% ES-grade Foetal Calf Serum (Invitrogen), 1\% L-glutamine (Gibco), 1\% non-essential amino acids (Gibco) and $0.0008 \% \beta$-mercaptoethanol (Sigma). The cells were kept at $37{ }^{\circ} \mathrm{C}$ in $5 \% \mathrm{CO}_{2}$ and $20 \% \mathrm{O}_{2}$ and cultured in gelatin-coated dishes or flasks $(0.1 \%$ gelatin, Sigma). MEF cell lines were kindly provided by the David Adams laboratory, Cambridge, UK and maintained in DMEM (high glucose, sodium pyruvate, Gibco) supplemented with $10 \%$ foetal bovine serum, $1 \%$ glutamine and $1 \%$ penicillin/streptomycin at $37^{\circ} \mathrm{C}$ in $5 \% \mathrm{CO}_{2}$ and $3 \% \mathrm{O}_{2}$. Mitomycin C (Sigma, 2 $\mathrm{mg}$ )-treated MEF SNL76/7 cells (obtained from ATCC) were used as the feeder layer for somatic reprogramming as well as adjusting E14Tg2a cells in feeder-free culture. Cells were routinely tested and found negative for mycoplasma infections.
\end{abstract}

Co-immunoprecipitation. For lysate preparation, $7 \times 10^{6}$ cells were lysed in icecold $1 \%$ (v/v) Nonidet P40 lysis buffer ( $20 \mathrm{mM}$ Hepes pH 7.5, $1 \%$ NP40 (Roche), $150 \mathrm{mM} \mathrm{NaCl}, 0.5 \mathrm{mM}$ EDTA, $50 \mathrm{mM} \mathrm{NaF}, 10 \mathrm{mM} \beta$-glycerophosphate, $0.5 \mathrm{mM}$ $\mathrm{Na}_{3} \mathrm{VO}_{4}$ and $1 \times$ EDTA free protease inhibitors (Roche)). Lysates were cleared by centrifugation at $4{ }^{\circ} \mathrm{C}$ at $10,000 \times g$ for $10 \mathrm{~min}$ and then were subject to sonication for $15 \mathrm{~s}$ at $20 \%$ amplitude. Samples were normalised with Bradford protein assay and equal volumes of protein $(1-2 \mathrm{mg})$ were used per reaction per experiment. Immunocomplexes were precipitated with Protein G sepharose (Sigma) or Protein G Dynabeads (Life Technologies). The antibodies used were: anti-YAP (H-125; sc15404), anti-YAP (clone 63.7;sc-101199), anti-TEF-4 (sc-67115). IPs were incubated on an end-to-end rotator for $3 \mathrm{~h}$ at $4{ }^{\circ} \mathrm{C}$ and, afterwards, the beads were washed three times with NP40 wash buffer. IP samples were resuspended in $50 \mu \mathrm{L}$ $1 \times$ Laemmli sample buffer ( $12.5 \%$ glycerol $(\mathrm{v} / \mathrm{v}), 2 \%$ SDS $(\mathrm{w} / \mathrm{v}), 78 \mathrm{mM}$ Tris $\mathrm{pH}$ 6.8, $720 \mathrm{mM} \beta$-mercaptoethanol, bromophenol blue) and boiled ( $\left.100^{\circ} \mathrm{C} ; 5 \mathrm{~min}\right)$.

Size exclusion chromatography. Whole cell extracts were prepared by resuspending frozen pellets in one packed-cell volume of buffer containing $10 \mathrm{mM}$ Tris$\mathrm{HCl}$ (pH 7.8), $200 \mathrm{mM} \mathrm{KCl}, 1 \mathrm{mg} / \mathrm{ml}$ of each protease inhibitor (pepstatin, aprotinin, chymostatin and leupeptin) and $1 \mathrm{mM}$ PMSF $1 \mathrm{mM}$ NEM. Five milligrams of protein in $200 \mu \mathrm{L}$ were filtered through $0.45-\mu \mathrm{m}$ cellulose acetate membrane and loaded onto a Superdex 200 HR 10/30 column (GE Healthcare, Little Chalfont, UK) prewashed with 2 column volumes of running buffer (20 mM HEPES pH 7.5, $150 \mathrm{mM} \mathrm{NaCl}, 0.5 \mathrm{mM}$ EDTA, $0.25 \mathrm{mM}$ DTT) and ran with the same buffer at a flow rate of $0.5 \mathrm{ml} / \mathrm{min}$. $0.25-\mathrm{ml}$ fractions were collected and 10 microlites of each fraction were analysed by Western blot. The column was calibrated with the following protein standards: thyroglobulin $(669 \mathrm{kDa})$, apoferritin $(443 \mathrm{kDa})$, alcohol dehydrogenase $(150 \mathrm{kDa})$, bovine serum albumin $(66 \mathrm{kDa})$, carbonic anhydrase $(29 \mathrm{kDa})$, and aprotinin $(6.5 \mathrm{kDa})$.
Immunoblot analysis. Input samples were lysed in Laemmli Lysis Buffer $(50 \mathrm{mM}$ Tris-HCl pH 6.8, 2\% SDS, $50 \mathrm{mM} \mathrm{NaF}, 10 \mathrm{mM} \beta$-glycerophosphate, $0.5 \mathrm{mM}$ $\mathrm{Na}_{3} \mathrm{VO}_{4}$ and $1 \times$ EDTA free protease inhibitors (Roche)) and normalised by NanoDrop (Thermo Scientific). Immunoprecipitates and cell lysates were western blotted as previously described ${ }^{65}$. The following primary antibodies were used at a concentration of 1:1000; TEF-4 (sc-67115), $\beta$-catenin (sc-7199), p73 (ep436Y), YAP (sc-15404 and sc-101199), Gapdh (abcam;2251-1; 1/10000), Tcf-3 (sc166411), FLAG (M2; Agilent; 200472-21), Rassfla (sc-58470), HA (Millipore;05904), Nanog (ab80892), Oct4 (ab19857), TEF-1 (BD Laboratories;610922), Tcf-4 (Cell Signaling; C48H11/2569), LATS1 (Bethyl Laboratories; A300-477A-1), pS127 YAP (Cell Signaling; 4911), pY357 YAP (ab62751), pSer (ab9332), GATA-1 (sc13053), MST2 (ab52641) and HRP conjugated anti-mouse and anti-rabbit secondary antibodies were used at a concentration of 1:5000 (Jackson Immunoresearch). Uncropped western blots of key experiments can be found in Supplementary Figs 9-11.

Mass spectrometry. Samples were prepared according to the coimmunoprecipitation protocol from whole cell lysates (see above) with a few modifications. Specifically, only Protein G Dynabeads (Life Technologies) were used to precipitate the proteins of interest. Moreover, after the $3 \mathrm{~h}$ incubation on the end-to-end rotator, the immunoprecipitates were washed four times, the two first being with NP40 lysis buffer and the last two only with filtered PBS. Finally, the tubes were left uncapped to air-dry for approximately $15 \mathrm{~min}$ and no Laemmli sample buffer was added to the immunoprecipitates. The samples were subject to mass spectrometry analysis using an Orbitrap mass spectrometer.

Methylation-specific polymerase chain reaction (PCR). The genomic DNA from pluripotent and differentiated mouse ESC was isolated using the Dneasy Blood and Tissue kit (Qiagen) according to the manufacturer's instructions. The methylation status of the Rassfia promoter region was determined by chemical modification of genomic DNA with sodium bisulfite and methylation-specific PCR Bisulfite treatment converts cytosine bases to uracil bases but has no effect on 5methylcytosine bases. The bisulfite-treated DNA was used as a template for the methylation-specific PCR reaction. Primers for the unmethylated DNA-specific reaction were: FW: 5'-GGTGTTGAAGTTGTGGTTTG-3' and REV: 5'-TATTATACCCAAAACAATACAC- $3^{\prime}$. Primers for the methylated DNA-specific reaction were: FW: $5^{\prime}$-TTTTGCGGTTTCGTTCGTTC- ${ }^{\prime}$ and REV: $5^{\prime}$-CCCGAAACGTACTACTATAAC- $3^{\prime 66}$.

qPCR analysis. Quantitative Real-Time PCR was done according to the Power SYBR Green Cells-to-Ct kit protocol (Applied Biosystems). After the production of CDNA, the cDNA was diluted down 2-4 times in Nuclease-free water. The PCR Cocktail was then assembled, containing the forward and reverse primers. Reactions were performed in 96-well plates (Life Technologies) and the PCR instrument (Applied Biosystems) was programmed as follows: Stage 1. Enzyme activation (hold), repeats 1 , temperature $95^{\circ} \mathrm{C}$, time $10 \mathrm{~min}$, Stage 2. PCR (cycle), repeats 40 , Step 1 . Temperature $95^{\circ} \mathrm{C}$, time $15 \mathrm{~s}$, Step 2 . Temperature $60^{\circ} \mathrm{C}$, time $1 \mathrm{~min}$, Stage 3. Dissociation curve, Step 1 . Temperature $95^{\circ} \mathrm{C}$, time $15 \mathrm{~s}$, Step 2 .

Temperature $60^{\circ} \mathrm{C}$, time $1 \mathrm{~min}$, Step 3 . Temperature $95^{\circ} \mathrm{C}$, time $30 \mathrm{~s}$, Step 4 .

Temperature $60^{\circ} \mathrm{C}$, time $15 \mathrm{~s}$. The further analysis of the results was done using the machine software. For qRT-PCR following ChIP and ChIP seq, the Input samples were at a concentration of $2.5 \mathrm{ng} / \mu \mathrm{L}$. The following primers were used: Oct4 FW: TGTGGACCTCAGGTTGGACT and REV: TTTCATGTCCTGGGACTCCTC, Nanog FW: AAGGATGAAGTGCAAGCGGT and REV: GGTGCTGAGCCC TTCTGAATC, Sox2 FW: CAGGAGTTGTCAAGGCAGAGA and REV: CTTAAGCCTCGGGCTCCAAA, Bmp4 FW: ACCAATGGAGCCATTCCGTA and REV: ACGACCATCAGCATTCGGTT, Vimentin FW: AGCAGTATGAAAGCGTGGCT and REV: CAGGGACTCGTTAGTGCCTTT, Gata4 FW: GCTCCATGTCCCAGACATTC and REV: ATGCATAGCCTTGTGGGGAC, Nestin FW: GAGGCGCTGGAACAGAGATT and REV: CACAGCCAGCTGGAACTTTTC, Rassfia FW: GTGGAGACTTCATCTGGGGC and REV:

Fig. 4 RASSF1A regulates LATS-mediated YAP phosphorylation and enforces differentiation entry via p73. a Subcellular localisation and expression of total YAP and pS127-YAP in gradually differentiating ESC cultures. pS127-YAP is significantly increased upon differentiation. $\mathbf{b}$ Size exclusion chromatography of ESC lysates via Gel filtration column and western blotting with indicated antibodies. c Phosphoproteomic analysis of YAP phosphorylation in RASSF1Aexpressing versus non-expressing ESC. All cells are transfected with human FLAG-YAP1. All mass-spec intensities were normalised to YAP intensities for each sample. See also Supplementary Fig 6a and Data 4. d Western blotting of YAP immunoprecipitates under indicated conditions for the indicated antibodies and quantification of p73 relative ratio to YAP. e Western blotting of FLAG immunoprecipitates from ESC transfected with the indicated constructs. FLAG-YAP mutants are triple-tagged. $\mathbf{f}$ Proximity ligation assay (PLA) demonstrates association of anti-pS127-YAP with anti-p73 antibodies. Red dots indicate positive association. The graph reports number of PLA events between p73/pS127 in the presence or absence of RASSF1A. See also Supplementary Fig 6b. $\mathbf{g}$ YAP ChIP on indicated differentiation-related gene promoters and $\mathbf{h}$ qPCR for germ layer-specific differentiation markers in ESC in response to indicated conditions. See also Supplementary Data 6. i ESC cultures expressing zsRASSF1A or empty vector (zsCtrl) were subject to neural differentiation assay via N2B27 and retinoic acid (RA). Scale bars: $25-50 \mu \mathrm{m}$. ${ }^{\star} P<0.05,{ }^{\star \star} P<0.01$ and ${ }^{\star \star \star} P<0.001$, respectively, of Student's $t$-test. Error bars indicate s.e.m. Data shown are representative of at least three independent experiments 
CAGCCTCATCCACGTTCGTA, Gapdh FW: CTCCACTCACGGCAAATTCA and REV: CGCTCCTGGAAGATGGTGAT, Sox1 FW: CTGCAGTA-

CAGCCCCATCTC and REV: CTTGACCAGAGATCCGAGGG, Pax3 FW: CAAACCCAAGCAGGTGACAA and REV: TTTACTCCTCAGGATGCGGC Oct4 promoter (Tead2 BS) FW: GCAGAAGGTCAGGTCCACTC and REV:

AGAACCCCACGACATCACTC, Oct4 promoter ( $\beta$-catenin BS) FW:

TGGGGGCAGAGAAGATGGTTG and REV: AAGGCAGCGACTTGGAAGCC, p53 upstream element FW: TGGGGCTAGAAGTACCTCCC and REV:
CGTGAGGGACACCATTTCCA, Fbxw7 upstream element FW: TTAGACTGGGATGGGAGGGG and REV: GGGTTTGTGGGGGAAGAGAG, Klf3 upstream element FW: TGGCGATTGTTGCTTGTTGG and REV: TCCCTCTCCTCTCTTCAGGC, Tead 1 upstream element FW: CCTTTTGCAGTGTTCCAGCC and REV: AGGGAAGGCTACTGAGAGGG, Rassf1A promoter FW: GTACAACACGCAATCCGTCA and REV: GAAGTCTCCACAGAGGTCGC, $\beta$-actin promoter FW: AAATGCTG-

CACTGTGCGGCG and REV: AGGCAACTTTCGGAACGGCG.

\begin{tabular}{c|cc|c|c|}
\begin{tabular}{c|c|c|} 
GEO: \\
Dataset
\end{tabular} & \multicolumn{2}{|c|}{$\begin{array}{c}\text { Oct4 inactivation } \\
\text { mESC } \\
\text { GDS3599 }\end{array}$} & \multicolumn{2}{|c}{$\begin{array}{c}\text { Brg1 depleted } \\
\text { 2cell embryo } \\
\text { GDS2156 }\end{array}$} \\
\hline Rassf1 & 1.56 & 0.01 & 1.44 & 0.045 \\
\hline Gata1 & 1.5 & 0.037 & - & 0.022 \\
\hline Cdkn1a & 1.91 & 0.00003 & 3.73 & 0.02 \\
Mdm2 & 1.92 & 0.001 & 1.7 & - \\
\hline Klf3 & 1.57 & 0.03 & SD & - \\
\hline Fbxw7 & 1.51 & 0.067 & 2.042 & 0.001 \\
\hline Nanog & 0.39 & 0.025 & ND & - \\
\hline
\end{tabular}

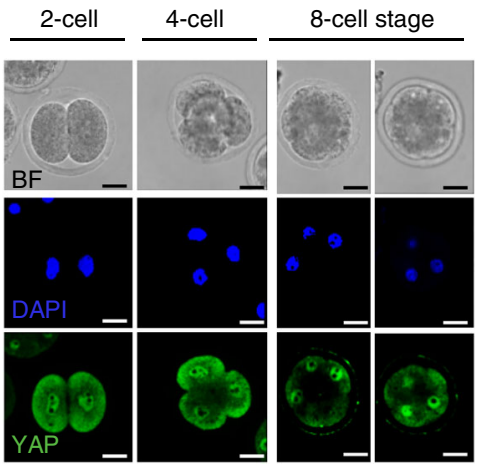

b

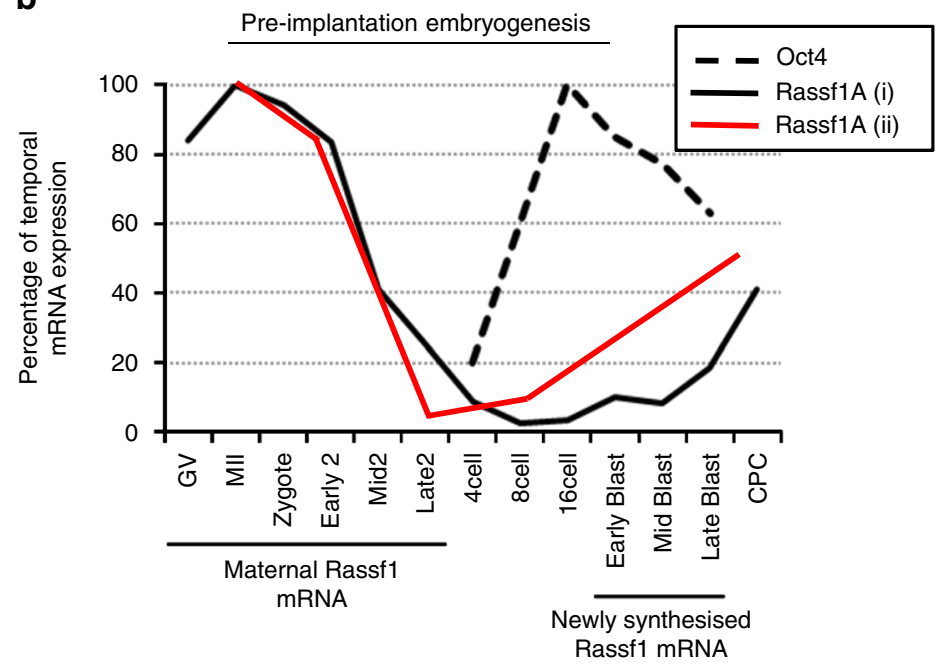

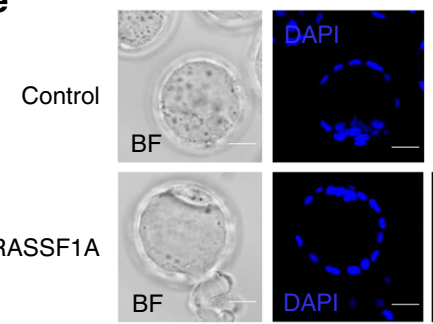
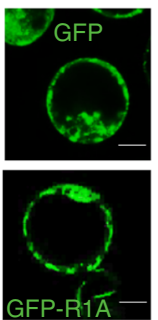

d

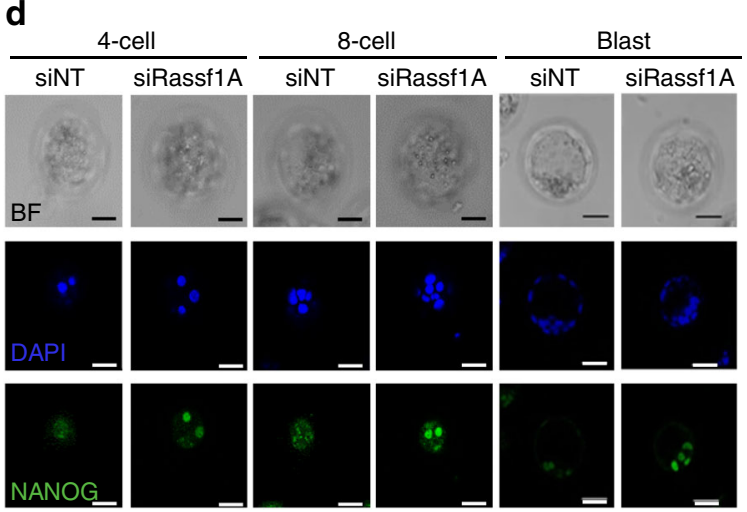

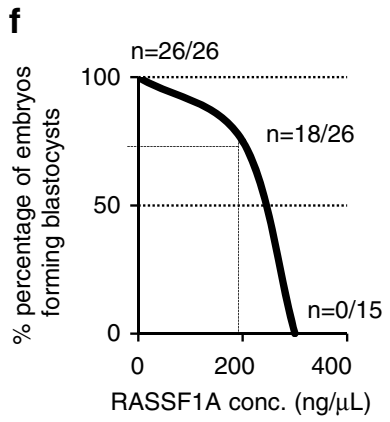

g

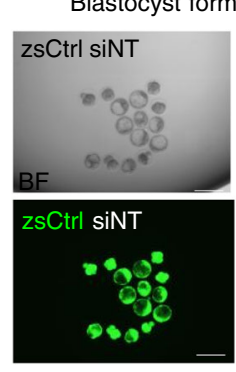

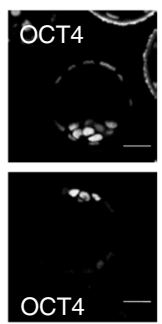

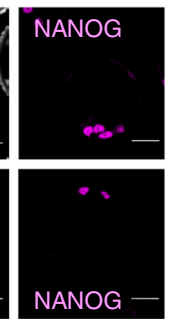

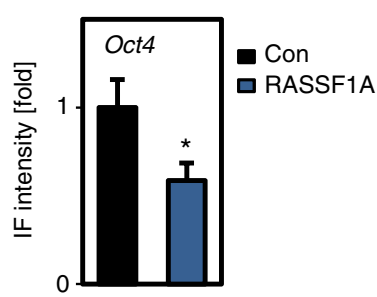


Immunofluorescence. Cells plated onto gelatin-coated glass coverslips (Fisher) were fixed in $4 \%$ Paraformaldehyde solution (in PBS), permeabilised with $0.2 \%$ Triton X solution (in PBS) and blocked in $0.2 \%$ fish skin gelatin (in PBS). The cells were next incubated in primary antibody at $4{ }^{\circ} \mathrm{C}$ overnight and then washed three times with PBS before secondary antibody incubation for $1 \mathrm{~h}$ at RT. Cells were mounted onto microscope slides using Prolong Gold antifade reagent with DAPI (Life Technologies) and confocal microscopy was carried out using Zeiss LSM710 microscope with the Zeiss ZEN software. The following primary (dilution 1:100) and secondary (dilution 1:500) antibodies were used: Nanog (ab80892), Oct4 (ab19857), Sox2 (MAB4423), pS127 YAP (Cell Signaling; 4911), p73 (Imgenex; img-246), ki67 (AB9260), YAP (sc-15404 and sc-101199), Nestin (sc-20978), Alexa fluor goat anti-rabbit and anti-mouse (Invitrogen).

DNA constructs and RNA interference experiments. For siRNA-mediated silencing and transient plasmid expression in ESCs, Lipofectamine 2000 (Invitrogen) was used in reverse transfections according to manufacturer's instructions. For plasmid expression in MEFs, electroporation was performed using the Nucleofector II device (Lonza). Infections were performed with the use of HEK293T cells. shRNA sequences were cloned into the pLKO.1 puro lentiviral vector to generate stable ESC lines. Stable MEF cell lines were generated after infection with a pBabe puro retroviral vector. The following oligonucleotides were used for siRNA-mediated silencing: non-targeting (NT): 5'-TAAGGTATGAAGAGATAC-3', Rassfla \#1 5' - CAGAACTCATTGAACTACGCGAGCT-3', Rassf1a \#2 5'-TGCGACCTCTGTGGAGACTTCATCT-3'. siRNAs to Tead2, Yap, Catnb, Trp73, Lats1, Tcf3, Tcf4, Gata1 and Tead1 were purchased from Qiagen. The following oligonucleotides were used for shRNA-mediated silencing: GFP 5'-CCGG-GCTGACCCTGAAGTTCATCTT-CTCGAG-AAGATGAACTTCAGGGTCAGC-TTTTTG-3' Rassfla 5'-CCGG-CTGCAAGTTCACCTGCCATTA-CTCGAG-TAATGGCAGGTGAACTTGCAG-TTTTTG-3', Tead2 5'CCGG-CCTCTTAGAAAGGAACCGGAA-CTCGAG-

TTCCGGTTCCTTTCTAAGAGG-TTTTTG-3'. MEFs transfected with pBabe puro were cultured in the presence of $1-2 \mu \mathrm{g} / \mathrm{mL}$ of puromycin for $2-3$ days. ESCs transfected with pLKO.1 puro were cultured in the presence of $1.75 \mu \mathrm{g} / \mathrm{mL}$ of puromycin for 2 days. pcDNA3 GATA1 was a gift from Licio Collavin \& Giannino Del Sal (Addgene plasmid \# 85693).

Proximity ligation assay (PLA). ESCs were transfected with the indicated constructs and $24 \mathrm{~h}$ post-transfection they were transferred into gelatinised $(0.1 \%$ gelatin) 16-well chamber slides (Fisher) and cultured for a further $24 \mathrm{~h}$. Cells were subsequently fixed and assayed using the PLA kit (Duolink) according to the manufacturer instructions. Briefly, after applying primary antibodies, cells were (1) Incubated with plus and minus probes for $1 \mathrm{~h},(2)$ DNA strands were subjected to ligase treatment for $30 \mathrm{~min}$, (3) DNA was amplified for $100 \mathrm{~min}$ with the addition of polymerase and (4) slides were mounted and PLA dots were detected via confocal microscopy (Zeiss LSM710), using the Zeiss ZEN software. The following primary antibodies were used: p73 (Imgenex; img-246; dilution 1:100) and pS127 YAP (Cell Signaling; 4911; dilution 1:100).

Reprogramming of MEFs into iPS cells. Reprogramming of MEFs was performed by inducing expression of defined transcription factors (Sox2, Klf4, Oct4, c-Myc, referred to as SKOM $)^{54}$. For transgene expression the piggyBac transposition system $^{55}$ was used. SKOM transposon was used together with PBase transposase. Immediately after the transfection, MEFs were seeded on feeder layer-containing dishes and left in culture for approximately 11 days before they were stained for Nanog and alkaline phosphatase expression using the Vector Black Alkaline Phosphatase Substrate Kit II.

Differentiation assays. For LIF withdrawal assays, ESCs and iPSCs originally cultured in complete ESC medium (ESC medium supplemented with LIF) were subject to LIF-free culture for 4-20 days and assessed periodically for morphological changes, expression of stem cell markers and differentiation capacity via microscopy, western blotting, qRT-PCR and differentiation assays. For EB formation, iPSCs were trypsinised and replated to $90 \mathrm{~mm}$ non-gelatinised Petri dishes (Sterilin) at a concentration of $4 \times 10^{5}$ cells/dish in a total volume of $20 \mathrm{ml} \mathrm{ESC}$ medium in the absence of LIF. The cells were cultured for 8 days, observing periodically to ensure that the medium did not become exhausted. Proliferation of stem cells under these conditions resulted in the formation of large aggregates that comprise EBs, the latter being macroscopic at approximately day $4-5$ of culture.

Differentiation towards neural progenitors was carried out as follows: EBs were allowed to be formed and then cultured in neural differentiation medium consisting of Neurobasal medium (Gibco 21103) supplemented with B27 (Gibco 17504), N2 (Invitrogen 17502-048) and RA (100 nM; Sigma R2625) for 3-4 days. Subsequently, EBs were stained for neural progenitor markers.

Differentiation towards DCs was carried out as previously described ${ }^{56}$. Briefly, after EB formation, all EBs were transferred to a $50 \mathrm{ml} \mathrm{Falcon}$ tube and allowed for 10-20 min to sediment under unit gravity. Following that, the medium was replaced with fresh ESC medium (LIF-free) prior to transferring to new Petri dishes. Six days later, EBs were harvested by sedimentation and washed gently twice in complete DMEM medium. The EBs were subsequently seeded at low density (approximately 10-30 EBs per plate) into non-gelatinised 10-cm tissue culture plates in LIF-free ESC medium supplemented with 2\% GM-CSFconditioned medium (Life Technologies) and $1000 \mathrm{U} / \mathrm{mL}$ of recombinant IL-3 (rIL-3;R \& D systems). Following overnight culture, a proportion of the EBs attached to the dishes and began to develop colonies in a radial fashion which continued to grow until they eventually formed a stromal layer. DCs were observed around the perimeter of the colonies approximately 4 days later and continued to expand.

Flow cytometry. Fixation and immunostaining of cells were done according to the Cell Signaling Technology protocol for Flow Cytometry. $10^{6}$ cells/condition were analysed on a BD FACSCalibur flow cytometer. A cd54 (I-CAM) antibody (Novus Biologicals; NBP1-72025; 1:100 dilution) was used.

Chromatin immunoprecipitation. ChIP from ESCs was performed according to the ChIP-IT High Sensitivity kit (Active Motif). Chromatin sonication was achieved with the use of Bioruptor Plus, Diagenode, at low amplitude, pulse for $30 \mathrm{~s}$ on and $30 \mathrm{~s}$ off for $20 \mathrm{~min}$ in ChIP buffer. 1/20 of the chromatin preparation was extracted to generate the Input. 15-30 $\mu$ g of chromatin and $4 \mu \mathrm{g}$ of antibody per ChIP reaction were used. Binding to promoter regions was assessed via qPCR. YAP (clone 63.7; sc-101199), TEF-4 (sc-67115), $\beta$-catenin (sc-7199), GATA-1 (sc13053), H3K4Me3 (ab8580), H3 (Cell Signalling; 14269 S), H3K27Me3 (Cell Signalling; 9733 S) and IgG (Cell Signalling; 2729 S) antibodies were used for the immunoprecipitations.

Embryo collection and culture. Animals were maintained in the Gurdon Institute Animal Facility at 12:12 light cycle and free access to food and water was constantly ensured. Experiments were conducted in accordance with Home Office regulations. For embryo collection, 4-6 weeks old mice belonging to the C57Bl6 and CBA strains ( $F_{1}$ generation) were superovulated with $10 \mathrm{IU}$ of pregnant mare serum gonadotropin (PMSG; Intervet) and $10 \mathrm{IU}$ human chorionic gonadotropin (Intervet) and mated with F1 $(\mathrm{C} 57 \mathrm{Bl} 6 \times \mathrm{CBA})$ males $48 \mathrm{~h}$ post treatment. Dissection of embryos out of the oviducts was performed into M2 medium and for zygote recovery cumulus cells were subjected to hyaluronidase treatment $(1 \mathrm{mg} / \mathrm{mL})$. Cultures of embryos were maintained in drops of KSOM media (Millipore) under paraffin oil in $5 \% \mathrm{CO}_{2}$ at $37^{\circ} \mathrm{C}$.

Embryo microinjection and analysis. Embryo microinjection was carried out on a Leica DM IRB microscope with attached Leica micromanipulators. Injections were carried out in embryos maintained in M2 medium under paraffin oil on a depression slide via air pressure with an Eppendorf Femtojet microinjector. Use of negative capacitance ensured membrane penetration.

For immunofluorescence experiments, embryos were fixed in 4\% PFA for 20 min and washed $2 \times 5 \mathrm{~min}$ in PBS-T (PBS containing $0.1 \%$ Tween).They were subsequently permeabilized with $0.5 \%$ Triton X-100 in PBS for 20 min and washed $3 \times 5 \mathrm{~min}$ in PBS-T. The following primary antibodies were used for overnight incubation following dilution in 3\% BSA (Sigma):Oct4 (Santa Cruz sc-5279; 1:200), Nanog (Abcam ab80892; 1:200) and Yap (Santa Cruz sc-15407; 1:400). Embryos were washed $3 \times 5 \mathrm{~min}$ in PBS-T before secondary antibodies (Invitrogen, AlexaFluor) and DAPI (Invitrogen) were applied for $1 \mathrm{~h}$ at a dilution of 1:400 and 1:1000 respectively in 3\% BSA (Sigma). Embryos were placed overnight into M2 media following $3 \times 5$ min washes in PBS-T and imaged on glass-bottom dishes to prevent flattening. Confocal images were acquired on a Leica SP5 microscope with

Fig. 5 Premature activation of RASSF1A impairs embryogenesis via p73. a Indicated gene expression levels in published GEO data sets GDS3599 and GDS2156. b Temporal expression of Oct4 and Rassf1A mRNA in the pre-implantation embryo (\% of maximum expression) from published GEO data sets GDS752 (black colour) and GDS814 (red colour). c Nuclear localisation of YAP during early stages of pre-implantation development. d Nanog immunofluorescence and e representative images of embryos microinjected with either control (zsCtrl) or RASSF1A-expressing (zsR1A) vectors stained for stem cell marker expression. Bar graph showing total OCT4 protein levels across all embryos in zsR1A versus zsCtrl. f 'Kill curve' to determine lethal RASSF1A concentration in pre-implantation embryos. The graph expresses percentage (\%) of blastocyst-forming embryos at the indicated RASSF1A concentration. $\mathbf{g}$ Viability of embryos in response to RASSF1A expression and/or sip73 microinjection, $n=15$. BF bright field channel. Scale bars: $10-50 \mu \mathrm{m}$. ${ }^{\star} P<0.05$, of Student's t-test. Error bars indicate s.e.m 
a

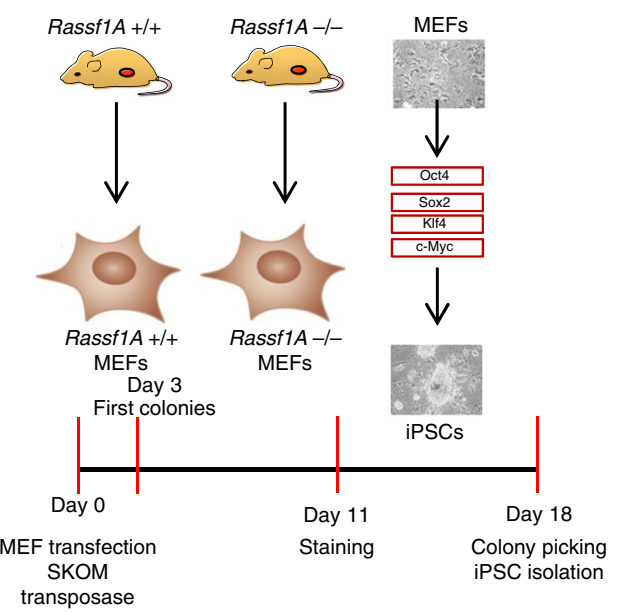

b

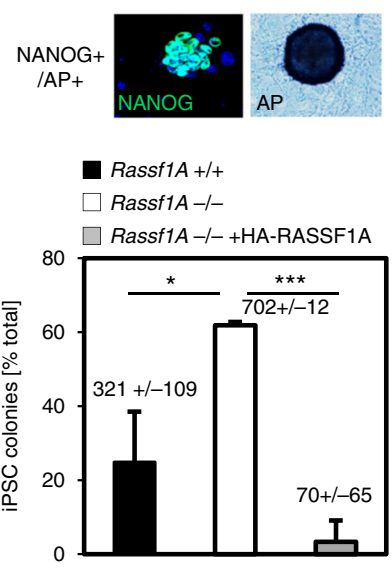

C

$R 1 A+/+$ MEFs

$R 1 A-/-$ MEFs

- $R 1 A+/+\mathrm{iPSCs}$

R1A-/-iPSCs

$\square R 1 A-/+$ HA-R1A iPSCs

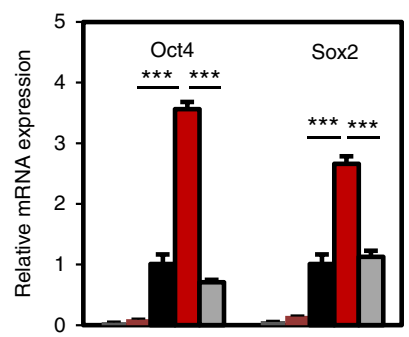

d

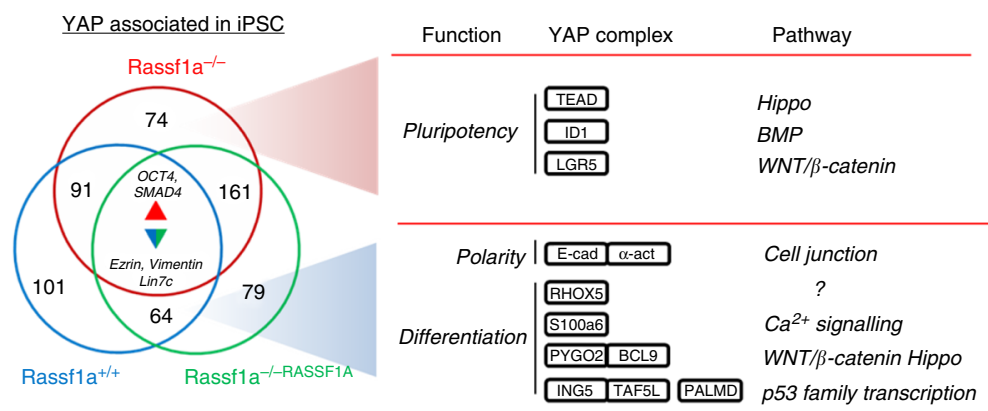

e

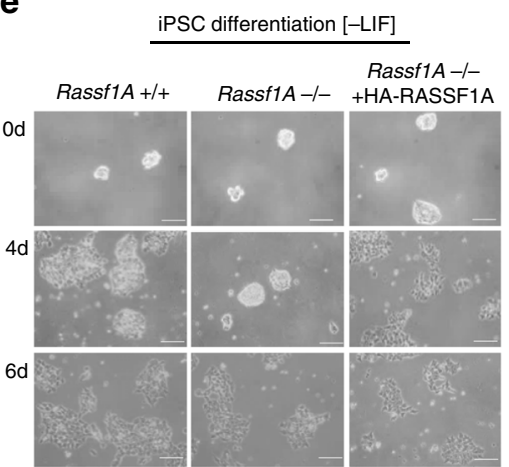

f

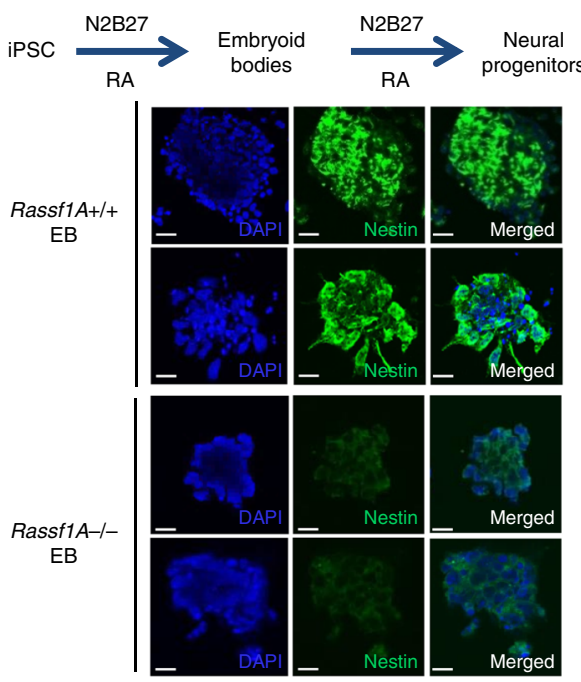

9

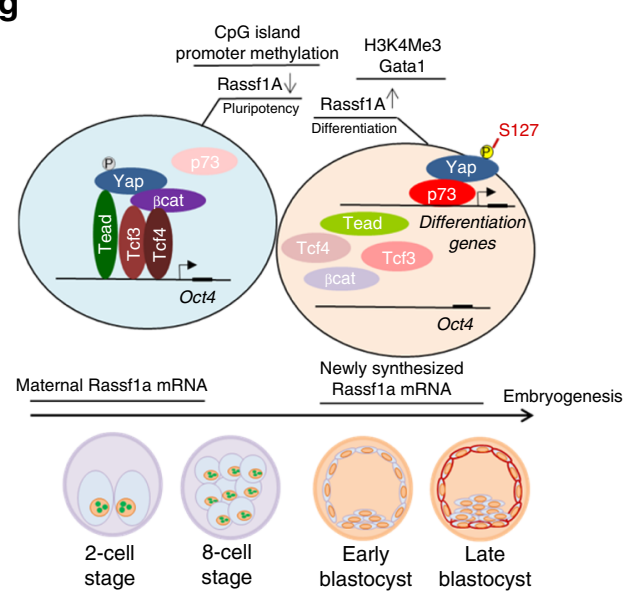

Fig. 6 RASSF1A is a barrier to somatic cell reprogramming and iPS cell self-renewal. a Experimental scheme for iPSC generation from MEFs. b Top: example images of Nanog/alkaline phosphatase (AP)-positive round iPSC colonies and quantification of reprogramming efficiency in the respective conditions. c qPCR in MEFs and iPSC for core stem cell marker expression. $\mathbf{d}$ Proteomics analysis for the YAP interactome in iPSC from indicated conditions. See also Supplementary Data 5. e Representative iPSC colonies from indicated conditions subject to LIF withdrawal assay. Quantification is provided in Supplementary Fig 8h. $\mathbf{f}$ Neural differentiation of Rassf1A ${ }^{+/+}$and ${ }^{-/-}$iPSC in N2B27 medium and retinoic acid (RA). Differentiation capacity of iPSC into neural progenitors is assessed via Nestin and Pax3 expression $\mathbf{g}$ Model. Scale bars: $25-50 \mu \mathrm{m}$. ${ }^{\star} P<0.05$, ${ }^{\star \star} P<0.01$ and ${ }^{\star \star \star} P<0.001$, respectively, of Student's $t$-test. Error bars indicate s.e.m. Data shown are representative of at least three independent experiments 
a Z-resolution of $4 \mu \mathrm{m}$. Signal intensity differences were layer normalised using IMARIS.

$z s C t r l$ and zsRASSF1A were cloned into pRN3p for in vitro transcription ${ }^{67}$. mRNA was produced using the mMESSAGE mMACHINE Kit (Life Technologies). RNA was extracted from embryos with the Arcturus PicoPure RNA Isolation Kit. qPCR was performed using SYBR Green in a StepOne Plus Real-time PCR machine (Applied Biosystems).

ChIPseq and RNAseq data processing. ChIP seq tags were pre-processed by trimGalore (http://www.bioinformatics.babraham.ac.uk/projects/trim_galore/) to remove sequencing adaptors and low quality regions. Trimmed read alignment was performed against mouse reference genome (mouse genome version $\mathrm{mm} 9$ ) with Bowtie aligner using default parameters. We used Picard tools to remove sequencing PCR duplicates. The ChIP seq peaks were identified independently by MACS2 (http://liulab.dfci.harvard.edu/MACS/) and $\mathrm{PePr}^{68}$ packages. The peaks called by both methods were subjected to further analyses. Both packages were run in narrow peaks detection mode and FDR threshold was 0.01 . RNA seq reads were pre-processed to remove sequencing adaptors and low quality regions as ChIP seq data. The resulting reads were aligned against reference genome by STAR aligner ${ }^{69}$. RNA seq raw read counts were calculated with htseq-count script using RefSeq gene annotation for mouse genome. The differential gene expression was called with DeSEQ2 R package. For integrative analysis of RNA seq and ChIP seq data, binding and expression data integration and analysis was performed by BETA software package in "plus" mode (http://cistrome.org/BETA/). The average profiles of ChIP enrichment signal within and near differentially expressed genes, as well as relative enrichment of ChIP regions in important genomic features were computed with CEAS package ${ }^{70}$

Additionally, FASTQC (http://www.bioinformatics.babraham.ac.uk) quality control was performed. FASTQC provides summary statistics and quality control checks on raw sequence data. Total sequences and sequence quality scores were considered as the main criteria, with default thresholds for rejection; when residual adaptor sequences were detected they were clipped using Trimmomatic (http:// www.usadellab.org). Mapping and alignment were done using Bowtie2 and TopHat2 (http://tophat.cbcb.umd.edu/). TopHat2 is a fast splice junction mapper for RNA sequencing reads; specifically, it aligns the reads to mammalian-sized genomes using the short read aligner Bowtie2, and then analyses the mapping results to identify splice junctions between exons. Paired pre- and post-treatment samples for each patient were processed using two different methods; Cuffdiff (http://cufflinks.cbcb.umd.edu) for transcript assembly and quantification of gene expression changes. Ensemble gene IDs were used as reference. Genes were ranked by their differential gene expression as estimated by Cuffdiff; rank product was used to merge samples from different conditions and to call the significantly upregulated and down-regulated genes; The Bioconductor EdgeR package (http:// www.bioconductor.org/packages/release/bioc/html/edgeR.html).

Statistical analysis. For all experiments reported in this manuscript, at least three biological replicates were used and statistical significance was determined by Student's $t$-test. To ensure randomization, for in vivo experiments animals were assigned randomly to either the experimental or control group. Additionally, the experiments were blinded to ensure unbiased interpretation of results.

Data availability statement. Published GEO data sets are analysed in Figs. 1, 5 and Supplementary Fig. 7. These have the following accession codes: In Fig. 1GSE54186, GSE3749, GDS2672; in Fig. 5-GDS3599, GDS2156, GDS752, GDS814 and GDS3599, GDS1824, GSE54186, GDS2156 were also discussed in Supplementary Fig. 7. The authors declare that all data supporting the findings of this study are available within the article and its supplementary information files or from the corresponding author upon reasonable request.

Received: 15 March 2017 Accepted: 29 December 2017

Published online: 30 January 2018

\section{References}

1. Boyer, L. A. et al. Core transcriptional regulatory circuitry in human embryonic stem cells. Cell 122, 947-956 (2005).

2. Tamm, C., Bower, N. \& Anneren, C. Regulation of mouse embryonic stem cell self-renewal by a Yes-YAP-TEAD2 signaling pathway downstream of LIF. J. Cell Sci. 124, 1136-1144 (2011).

3. Kaneko, K. J., Cullinan, E. B., Latham, K. E. \& DePamphilis, M. L. Transcription factor mTEAD-2 is selectively expressed at the beginning of zygotic gene expression in the mouse. Development 124, 1963-1973 (1997).

4. Sasaki, H. Roles and regulations of Hippo signaling during preimplantation mouse development. Dev. Growth Differ. 59, 12-20 (2017).

5. Lian, I. et al. The role of YAP transcription coactivator in regulating stem cell self-renewal and differentiation. Genes Dev. 24, 1106-1118 (2010).
6. Qin, H. et al. YAP Induces Human Naive Pluripotency. Cell Rep. 14, 2301-2312 (2016).

7. Huang, Z. et al. YAP stabilizes SMAD1 and promotes BMP2-induced neocortical astrocytic differentiation. Development 143, 2398-2409 (2016).

8. Lee, D. H. et al. LATS-YAP/TAZ controls lineage specification by regulating TGFbeta signaling and Hnf4alpha expression during liver development. Nat. Commun. 7, 11961 (2016).

9. Hong, J. H. et al. TAZ, a transcriptional modulator of mesenchymal stem cell differentiation. Science 309, 1074-1078 (2005).

10. Tang, Y., Feinberg, T., Keller, E. T., Li, X. Y. \& Weiss, S. J. Snail/Slug binding interactions with YAP/TAZ control skeletal stem cell self-renewal and differentiation. Nat. Cell Biol. 18, 917-929 (2016).

11. Sato, N., Meijer, L., Skaltsounis, L., Greengard, P. \& Brivanlou, A. H. Maintenance of pluripotency in human and mouse embryonic stem cells through activation of Wnt signaling by a pharmacological GSK-3-specific inhibitor. Nat. Med. 10, 55-63 (2004).

12. Ying, Q. L. et al. The ground state of embryonic stem cell self-renewal. Nature 453, 519-523 (2008).

13. Heallen, T. et al. Hippo pathway inhibits Wnt signaling to restrain cardiomyocyte proliferation and heart size. Science 332, 458-461 (2011).

14. Meng, Z., Moroishi, T. \& Guan, K. L. Mechanisms of Hippo pathway regulation. Genes Dev. 30, 1-17 (2016).

15. Wang, Q. et al. The p53 Family Coordinates Wnt and Nodal Inputs in Mesendodermal Differentiation of Embryonic Stem Cells. Cell Stem Cell 20, 70-86 (2017).

16. Fernandez-Alonso, R. et al. p73 is required for endothelial cell differentiation, migration and the formation of vascular networks regulating VEGF and TGFbeta signaling. Cell Death Differ. 22, 1287-1299 (2015).

17. Agostini, M. et al. Neuronal differentiation by TAp73 is mediated by microRNA-34a regulation of synaptic protein targets. Proc. Natl. Acad. Sci. USA 108, 21093-21098 (2011).

18. Fontemaggi, G. et al. Identification of direct p73 target genes combining DNA microarray and chromatin immunoprecipitation analyses. J. Biol. Chem. 277, 43359-43368 (2002).

19. Donninger, H. et al. Salvador protein is a tumor suppressor effector of RASSF1A with hippo pathway-independent functions. J. Biol. Chem. 286, 18483-18491 (2011).

20. Kawahara, M. et al. Kpm/Lats2 is linked to chemosensitivity of leukemic cells through the stabilization of p73. Blood 112, 3856-3866 (2008).

21. Matallanas, D. et al. RASSF1A elicits apoptosis through an MST2 pathway directing proapoptotic transcription by the 73 tumor suppressor protein. Mol. Cell 27, 962-975 (2007).

22. Oka, T., Mazack, V. \& Sudol, M. Mst2 and Lats kinases regulate apoptotic function of Yes kinase-associated protein (YAP). J. Biol. Chem. 283, 27534-27546 (2008)

23. Hamilton, G., Yee, K. S., Scrace, S. \& O'Neill, E. ATM regulates a RASSF1Adependent DNA damage response. Curr. Biol. 19, 2020-2025 (2009).

24. van der Weyden, L. et al. Loss of RASSF1A synergizes with deregulated RUNX2 signaling in tumorigenesis. Cancer Res. 72, 3817-3827 (2012).

25. Grawenda, A. M. \& O'Neill, E. Clinical utility of RASSF1A methylation in human malignancies. Br. J. Cancer 113, 372-381 (2015).

26. Vlahov, N. et al. Alternate RASSF1 Transcripts Control SRC Activity, ECadherin Contacts, and YAP-Mediated Invasion. Curr. Biol. 25, 3019-3034 (2015).

27. Pefani, D. E. et al. TGF-beta Targets the Hippo Pathway Scaffold RASSF1A to Facilitate YAP/SMAD2 Nuclear Translocation. Mol. Cell 63, 156-166 (2016).

28. Teng, I. W. et al. Targeted methylation of two tumor suppressor genes is sufficient to transform mesenchymal stem cells into cancer stem/initiating cells. Cancer Res. 71, 4653-4663 (2011).

29. Ben-Porath, I. et al. An embryonic stem cell-like gene expression signature in poorly differentiated aggressive human tumors. Nat. Genet. 40, 499-507 (2008).

30. Hochedlinger, K., Yamada, Y., Beard, C. \& Jaenisch, R. Ectopic expression of Oct-4 blocks progenitor-cell differentiation and causes dysplasia in epithelial tissues. Cell 121, 465-477 (2005).

31. Smith, Z. D. et al. DNA methylation dynamics of the human preimplantation embryo. Nature 511, 611-615 (2014).

32. Leung, C. Y. \& Zernicka-Goetz, M. Angiomotin prevents pluripotent lineage differentiation in mouse embryos via Hippo pathway-dependent and independent mechanisms. Nat. Commun. 4, 2251 (2013).

33. Salvianti, F. et al. Prospective evaluation of RASSF1A cell-free DNA as a biomarker of pre-eclampsia. Placenta 36, 996-1001 (2015).

34. Buschbeck, M. \& Hake, S. B. Variants of core histones and their roles in cell fate decisions, development and cancer. Nat. Rev. Mol. Cell Biol. 18, 299-314 (2017).

35. Meissner, A. et al. Genome-scale DNA methylation maps of pluripotent and differentiated cells. Nature 454, 766-770 (2008). 
36. Fujikura, J. et al. Differentiation of embryonic stem cells is induced by GATA factors. Genes Dev. 16, 784-789 (2002).

37. Bai, H., Sakurai, T., Godkin, J. D. \& Imakawa, K. Expression and potential role of GATA factors in trophoblast development. J. Reprod. Dev. 59, 1-6 (2013).

38. Beuling, E. et al. GATA factors regulate proliferation, differentiation, and gene expression in small intestine of mature mice. Gastroenterology 140, 1219-1229 (2011).

39. Beyer, T. A. et al. Switch enhancers interpret TGF-beta and Hippo signaling to control cell fate in human embryonic stem cells. Cell Rep. 5, 1611-1624 (2013).

40. Yi, F. et al. Opposing effects of Tcf3 and Tcf1 control Wnt stimulation of embryonic stem cell self-renewal. Nat. Cell Biol. 13, 762-770 (2011).

41. Behrens, J. et al. Functional interaction of beta-catenin with the transcription factor LEF-1. Nature 382, 638-642 (1996).

42. Zhao, B. et al. Inactivation of YAP oncoprotein by the Hippo pathway is involved in cell contact inhibition and tissue growth control. Genes Dev. 21, 2747-2761 (2007).

43. Hong, A. W. et al. Osmotic stress-induced phosphorylation by NLK at Ser128 activates YAP. EMBO Rep. 18, 72-86 (2017).

44. Mao, J. H. et al. Fbxw7/Cdc4 is a p53-dependent, haploinsufficient tumour suppressor gene. Nature 432, 775-779 (2004).

45. Zeron-Medina, J. et al. A polymorphic p53 response element in KIT ligand influences cancer risk and has undergone natural selection. Cell 155, 410-422 (2013).

46. Liu, H. et al. p53 regulates neural stem cell proliferation and differentiation via BMP-Smad1 signaling and Id1. Stem Cells Dev. 22, 913-927 (2013).

47. He, C. et al. c-myc in the hematopoietic lineage is crucial for its angiogenic function in the mouse embryo. Development 135, 2467-2477 (2008).

48. Castro, I. C. et al. MYC-induced epigenetic activation of GATA4 in lung adenocarcinoma. Mol. Cancer Res. 11, 161-172 (2013).

49. Aksoy, I. et al. Klf4 and Klf5 differentially inhibit mesoderm and endoderm differentiation in embryonic stem cells. Nat. Commun. 5, 3719 (2014).

50. Wang, Q. T. et al. A genome-wide study of gene activity reveals developmental signaling pathways in the preimplantation mouse embryo. Dev. Cell 6, 133-144 (2004).

51. Zeng, F., Baldwin, D. A. \& Schultz, R. M. Transcript profiling during preimplantation mouse development. Dev. Biol. 272, 483-496 (2004).

52. Masui, S. et al. Pluripotency governed by Sox 2 via regulation of Oct $3 / 4$ expression in mouse embryonic stem cells. Nat. Cell Biol. 9, 625-635 (2007).

53. Lee, M. T. et al. Nanog, Pou5f1 and SoxB1 activate zygotic gene expression during the maternal-to-zygotic transition. Nature 503, 360-364 (2013).

54. Takahashi, K. \& Yamanaka, S. Induction of pluripotent stem cells from mouse embryonic and adult fibroblast cultures by defined factors. Cell 126, 663-676 (2006).

55. Woltjen, $\mathrm{K}$. et al. piggyBac transposition reprograms fibroblasts to induced pluripotent stem cells. Nature 458, 766-770 (2009).

56. Fairchild, P. J. et al. Directed differentiation of dendritic cells from mouse embryonic stem cells. Curr. Biol. 10, 1515-1518 (2000).

57. Lien, W. H. \& Fuchs, E. Wnt some lose some: transcriptional governance of stem cells by Wnt/beta-catenin signaling. Genes Dev. 28, 1517-1532 (2014).

58. Messerschmidt, D. M., Knowles, B. B. \& Solter, D. DNA methylation dynamics during epigenetic reprogramming in the germline and preimplantation embryos. Genes Dev. 28, 812-828 (2014).

59. Fang, X., Corrales, J., Thornton, C., Scheffler, B. E. \& Willett, K. L. Global and gene specific DNA methylation changes during zebrafish development. Comp. Biochem. Physiol. B Biochem. Mol. Biol. 166, 99-108 (2013).

60. Kim, M. J. et al. Association of fetal-derived hypermethylated RASSF1A concentration in placenta-mediated pregnancy complications. Placenta 34, 57-61 (2013).

61. Fraga, L. R. et al. Interaction between TP63 and MDM2 genes and the risk of recurrent pregnancy loss. Eur. J. Obstet. Gynecol. Reprod. Biol. 182, 7-10 (2014).

62. Morin-Kensicki, E. M. et al. Defects in yolk sac vasculogenesis, chorioallantoic fusion, and embryonic axis elongation in mice with targeted disruption of Yap65. Mol. Cell Biol. 26, 77-87 (2006).
63. Zhang, X. et al. Analysis of liver tumor-prone mouse models of the hippo kinase scaffold proteins RASSF1A and SAV1. Cancer Res. 76, 2824-2835 (2016).

64. Park, J. et al. Tumor suppressor ras association domain family 5 (RASSF5/ NORE1) mediates death receptor ligand-induced apoptosis. J. Biol. Chem. 285, 35029-35038 (2010)

65. Pefani, D. E. et al. RASSF1A-LATS1 signalling stabilizes replication forks by restricting CDK2-mediated phosphorylation of BRCA2. Nat. Cell Biol. 16, 962-971 (2014).

66. Tommasi, S. et al. Tumor susceptibility of Rassfla knockout mice. Cancer Res. 65, 92-98 (2005).

67. Zernicka-Goetz, M. et al. Following cell fate in the living mouse embryo. Development 124, 1133-1137 (1997).

68. Zhang, Y., Lin, Y. H., Johnson, T. D., Rozek, L. S. \& Sartor, M. A. PePr: a peakcalling prioritization pipeline to identify consistent or differential peaks from replicated ChIP-Seq data. Bioinformatics 30, 2568-2575 (2014).

69. Dobin, A. et al. STAR: ultrafast universal RNA-seq aligner. Bioinformatics 29, 15-21 (2013).

70. Shin, H., Liu, T., Manrai, A. K. \& Liu, X. S. CEAS: cis-regulatory element annotation system. Bioinformatics 25, 2605-2606 (2009).

\section{Acknowledgements}

We thank L. van der Weyden for MEFs and Dr P. Liu for his valuable input regarding somatic cell reprogramming. We also thank Prof Hans Clevers, Prof Colin Goding, Dr Skirmantas Kriaucionis and Dr Vincenzo D'Angiolella for reading this manuscript. This work is supported by CRUK A19277, MRC, Pancreatic Cancer UK, the Federal Agency for Scientific Organizations (FASO Russia grant $N^{\circ} 0324-2016-0008$ ), the Wellcome Trust and the Science Foundation Ireland (grant $\mathrm{N}^{\circ} 15 / \mathrm{CDA} / 3495$ ).

\section{Author contributions}

A.P. and E.O.N. designed the study, designed and performed experiments, analysed and interpreted data. L.B., A.T., C.Y.L., K.T., D.K., D-E.P., C.R., C.G. and A.v.K. performed experiments and analysed data. A.G., S.H., J.C., A.K., F.B., D.M. and A.S. analysed data. G.H., N.V. and L.B. contributed to research tools. X.L., G.D., D.M. and M.Z-G. also helped with the study design and data interpretation. A.P. and E.O.N. wrote the manuscript and E.O.N. supervised the project.

\section{Additional information}

Supplementary Information accompanies this paper at https://doi.org/10.1038/s41467017-02786-5.

Competing interests: The authors declare no competing financial interests.

Reprints and permission information is available online at http://npg.nature.com/ reprintsandpermissions/

Publisher's note: Springer Nature remains neutral with regard to jurisdictional claims in published maps and institutional affiliations.

\section{(i)}

Open Access This article is licensed under a Creative Commons Attribution 4.0 International License, which permits use, sharing, adaptation, distribution and reproduction in any medium or format, as long as you give appropriate credit to the original author(s) and the source, provide a link to the Creative Commons license, and indicate if changes were made. The images or other third party material in this article are included in the article's Creative Commons license, unless indicated otherwise in a credit line to the material. If material is not included in the article's Creative Commons license and your intended use is not permitted by statutory regulation or exceeds the permitted use, you will need to obtain permission directly from the copyright holder. To view a copy of this license, visit http://creativecommons.org/ licenses/by/4.0/

(C) The Author(s) 2018 